\title{
Dengue virus-elicited tryptase induces endothelial permeability and shock
}

\author{
Abhay P.S. Rathore, ${ }^{1}$ Chinmay Kumar Mantri, ${ }^{1}$ Siti A.B. Aman, ${ }^{1}$ Ayesa Syenina, ${ }^{1}$ Justin Ooi, ${ }^{1}$ Cyril J. Jagaraj, ${ }^{1}$ Chi Ching Coh, ${ }^{2,3}$ \\ Hasitha Tissera, ${ }^{4}$ Annelies Wilder-Smith, ${ }^{5}$ Lai Guan Ng, ${ }^{2,3,6}$ Duane J. Gubler, ${ }^{1}$ and Ashley L. St. John ${ }^{1,6,7}$ \\ 1Program in Emerging Infectious Diseases, Duke-National University of Singapore, Singapore. ${ }^{2}$ Singapore Immunology Network (SlgN), Agency for Science, Technology and Research (A*STAR), Singapore. \\ ${ }^{3}$ School of Biological Sciences, Nanyang Technological University, Singapore. ${ }^{4}$ Epidemiology Unit, Ministry of Health and National Dengue Control Unit, Colombo, Sri Lanka. ${ }^{5}$ Lee Kong Chian School of \\ Medicine, Nanyang Technological University, Singapore. ${ }^{6}$ Department of Microbiology and Immunology, Yong Loo Lin School of Medicine, National University of Singapore, Singapore. ${ }^{7}$ Department of \\ Pathology, Duke University Medical Center, Durham, North Carolina, USA.
}

\begin{abstract}
Dengue virus (DENV) infection causes a characteristic pathology in humans involving dysregulation of the vascular system. In some patients with dengue hemorrhagic fever (DHF), vascular pathology can become severe, resulting in extensive microvascular permeability and plasma leakage into tissues and organs. Mast cells (MCs), which line blood vessels and regulate vascular function, are able to detect DENV in vivo and promote vascular leakage. Here, we showed that an MC-derived protease, tryptase, is consequential for promoting vascular permeability during DENV infection through inducing breakdown of endothelial cell tight junctions. Injected tryptase alone was sufficient to induce plasma loss from the circulation and hypovolemic shock in animals. A potent tryptase inhibitor, nafamostat mesylate, blocked DENV-induced vascular leakage in vivo. Importantly, in 2 independent human dengue cohorts, tryptase levels correlated with the grade of DHF severity. This study defines an immune mechanism by which DENV can induce vascular pathology and shock.
\end{abstract}

\section{Introduction}

In humans, dengue virus (DENV) causes an acute viral infection that manifests as a broad spectrum of disease: from asymptomatic infection to a mild febrile illness, dengue fever (DF), which may resolve within 2 weeks to the most severe form, dengue hemorrhagic fever (DHF), which involves vascular leakage as the primary potentially life-threatening sign. When homeostatic mechanisms fail, the widespread vascular leakage that occurs during DHF can also lead to dengue shock syndrome (DSS) resulting from hypovolemia $(1,2)$. In the initial classification scheme for DHF/DSS, patients were also graded according to the severity of DHF: DHF-I, involving fever accompanied by positive tourniquet test and or easy bruising; DHF-II, involving spontaneous bleeding or frank hemorrhaging; DHF-III, characterized by shock, such as circulatory failure due to rapid weak pulse and narrowing of pulse pressure; and DHF-IV, characterized by severe shock without a pulse that is usually fatal (3). The predominant view of the field is that vascular leakage in humans results from immune-mediated pathology rather than infection of endothelial cells themselves (2). One theory is that "cytokine storm" damages the vascular endothelium during infection. These cytokines could be derived from infected cells that contain replicating virus or other immune cells

Related Commentary: p. 4072

Conflict of interest: The authors have declared that no conflict of interest exists. Copyright: (5) 2019, American Society for Clinical Investigation.

Submitted: February 26, 2019; Accepted: June 25, 2019; Published: August 26, 2019.

Reference information: J Clin Invest. 2019;129(10):4180-4193.

https://doi.org/10.1172/JCI128426. that respond to infection, such as T cells and mast cells (MCs) (4, 5). TNF, for example, has been shown to promote vascular leakage and death due to dengue in an immunocompromised mouse model (6); however, in human patients, there have been less clear associations between specific vasoactive cytokines and vascular leakage during infection and even conflicting associations in various studies $(2,7-9)$. If cytokine storm is involved, it is also unclear why symptoms of dengue differ from other conditions that involve cytokine storm (2). Others have identified high levels of complement activation in pediatric DSS patients (10) that could be further enhanced in the presence of NS1 protein (11). Reports suggest that NS1 alone can directly induce vascular permeability $(12,13)$, and there are conflicting reports regarding whether NS1 levels correlate with DENV disease severity in human studies as well as in animal models (14-18). Thus, we do not fully understand the mechanism of DENV-induced vascular leakage in humans. Even in mild dengue cases, usually diagnosed as DF, signs and symptoms of dengue vascular pathogenesis and immune activation can be present, such as bruising, purpura, petechiae, rash, and hemoconcentration $(1,2)$. It is also possible that the signs of vascular leakage, hemorrhaging, and shock are not merely due to an augmentation of the same mechanism, but may have independent mechanisms that contribute to the spectrum of disease.

Interestingly, some clinical signs similar to those that occur as a result of DENV infection have been associated with the effects of activated MCs in independent clinical contexts, such as during allergic reactions or anaphylactic shock (19). These include signs such as vascular leakage, rash, flushing, and abdominal pain (20, 21). In those allergic conditions, MC-derived products can cause urticaria, edema, vasodilation, and blood pressure changes (19). 
Mature MCs do not circulate in the blood, but are found only in connective and mucosal tissues (22). There they act as sentinels for pathogens, including $\operatorname{DENV}(23,24)$, and also serve an immune-regulatory function at tissue sites (25). In addition to their relatively even dispersal throughout the skin and mucosae, a portion of MCs also adopt a perivascular distribution in vivo (26). It has been shown in mice that MCs may even extend processes into the lumen of blood vessels (27). They store vasoactive mediators in their granules, including heparin, tryptase, chymase, additional proteases, and preformed cytokines (e.g., TNF) (26, 28). MC granules remain insoluble for hours after their release as membrane-free particles, and they are thought to slowly release their cargo in the extracellular environment $(29,30)$. Proteases account for the majority of the granule protein content, of which chymase and tryptase are the most abundant (28). Chymase is understood to affect the renin-angiotensin system as an angiotensin I-to-angiotensin II-converting enzyme (31), while tryptase has primarily been characterized as playing a role in vascular leakage through its ability to cleave protease-activated receptor (PARs) (32).

In vivo studies revealed that MCs play a key role in protective immune response involving cellular recruitment to sites of localized cutaneous DENV infection in mice (24). However, as the infection spreads and becomes systemic, the role of MCs becomes more complex (4). We previously investigated the interactions between MCs and DENV in the mouse model and determined that DENV induces MC degranulation (24). Mature MCs are highly resistant to infection, and degranulation occurs independently of virus replication within MCs, since UV-inactivated virus triggers a degranulation response similar to live virus (24). MCs also promoted recruitment of $\mathrm{T}$ and NK cells to infection sites and viral clearance through de novo chemokine production (24) and activation of cytotoxic T cells, such as $\gamma \delta \mathrm{T}$ cells, through nonclassical antigen presentation (33). Contrasting with their protective function in localized infection, widespread activation of MCs during systemic infection can contribute to DENV-induced vascular leakage in mice (34). Antibodies can also enhance the DENV-induced activation of MCs by crosslinking of activating Fc $\gamma$ Rs, similar to the mechanism described for MC activation during the reverse arthus reaction $(5,35)$. In human DENV patients, detection of heightened serum protein levels of the MC-specific and granule-associated product, chymase, during the acute febrile phase of disease indicated that MCs are activated during DENV infection $(34,36)$. Chymase was not elevated in the serum of other febrile patients, most of which were experiencing respiratory infections (34). Importantly, chymase levels were higher in the serum of patients diagnosed with DHF than those diagnosed with DF during both the acute febrile (within 3 days of fever onset in that study) and defervescent phases of disease, but were highest at the earliest time points and higher in DHF/DSS patients experiencing secondary infections compared with those experiencing primary infection (34). Thus, chymase levels were correlated with disease severity prior to diagnosis of severe disease, raising the possibility that chymase could serve as a prognostic biomarker for severe dengue $(34,36)$. Taken together, the data suggest that systemic DENV infection initiates a cascade of events involving widespread MC activation, leading to persistent levels of MC-derived products in the serum, which damage the vascular endothelium, throughout the acute phase of disease. Yet the mechanism by which MCs promote endothelial activation and vascular permeability during DENV infection remains unknown. In this study, we sought to identify the MC mediator primarily responsible for endothelial breakdown and vascular leak during DENV disease. We show that DENV-elicited tryptase is a particularly potent inducer of microvascular permeability, which occurs mechanistically through disruption of endothelial tight junctions. Validation of these findings in mice suggests that tryptase promotes substantial increases in plasma loss from the circulation and reduced adhesion molecule expression on vascular endothelial cells, while chymase, in contrast, has a more modest effect. A potent specific inhibitor against tryptase was sufficient to reduce DENV-induced vascular leak in vivo in multiple mouse models. Remarkably, in human patients with DHF, levels of serum tryptase were highest when patients experienced shock (DHF grades III-IV) and were well correlated with the severity of DHF. These findings suggest that high levels of tryptase can cause shock during DHF.

\section{Results}

$M C$ proteases tryptase and chymase promote endothelial permeability and break tight junctions. To identify the MC-derived products that promote vascular leakage during DENV infection, we first questioned whether the soluble or particulate (exocytosed MC granules; ref. 30) fractions were primarily responsible for inducing endothelial permeability. The soluble fraction of media after MC activation would contain the de novo synthesized products, such as eicosanoids and most cytokines, as well as prestored mediators that are quickly solubilized from the granule after activation, such as histamine. The particulate fraction would contain largely the insoluble prestored mediators, including heparin, and the proteases that make up the majority of heparin-associated proteins, including chymase and tryptase (37). These fractions were separated by centrifugation, and human microvascular endothelial cells (huMECs) were cultured to form a tight monolayer, followed by treatment of the monolayers with total or fractionated portions of DENV-induced MC products. The degree of damage to the endothelial integrity was determined by measuring transendothelial resistance (TER). Our previous studies showed that the TER was dramatically reduced when endothelial monolayers were treated with DENV-elicited MC products compared with the control untreated groups, groups treated with supernatants from mock-infected MCs, or groups treated with an equivalent amount of DENV in media alone (34). Here, when DENV-elicited MC products were further separated into soluble and particulate fractions, treatment of huMEC monolayers with the particulate fraction significantly reduced TER compared with the soluble fraction treatment group, while the same concentration of virus alone did not have a significant effect (Figure 1A). These data suggest that it is the MC granule-associated components that are important in breaking endothelial integrity.

The two dominant protein constituents of granules are the MC proteases chymase and tryptase. We previously had determined that chymase is a biomarker of severe vascular leakage in human DENV patients (34), but whether it also has a functional role in DENVinduced vascular leakage remained unclear. In order to address the direct ability of MC proteases to induce endothelial permeability, we cloned, expressed, and purified the two dominant MC proteases, 
A

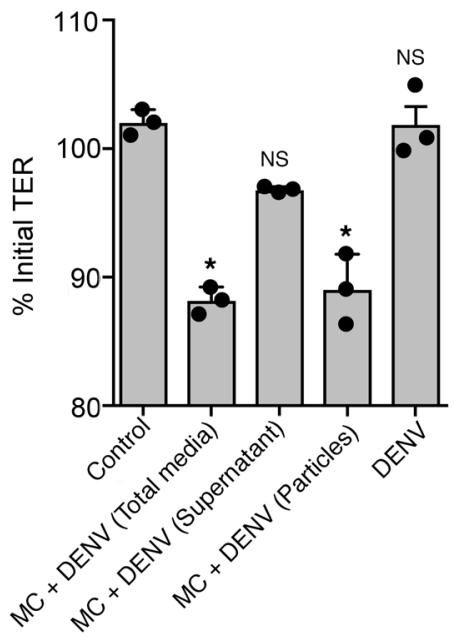

D Tubulin

$\mathrm{ZO}-1$

DAPI
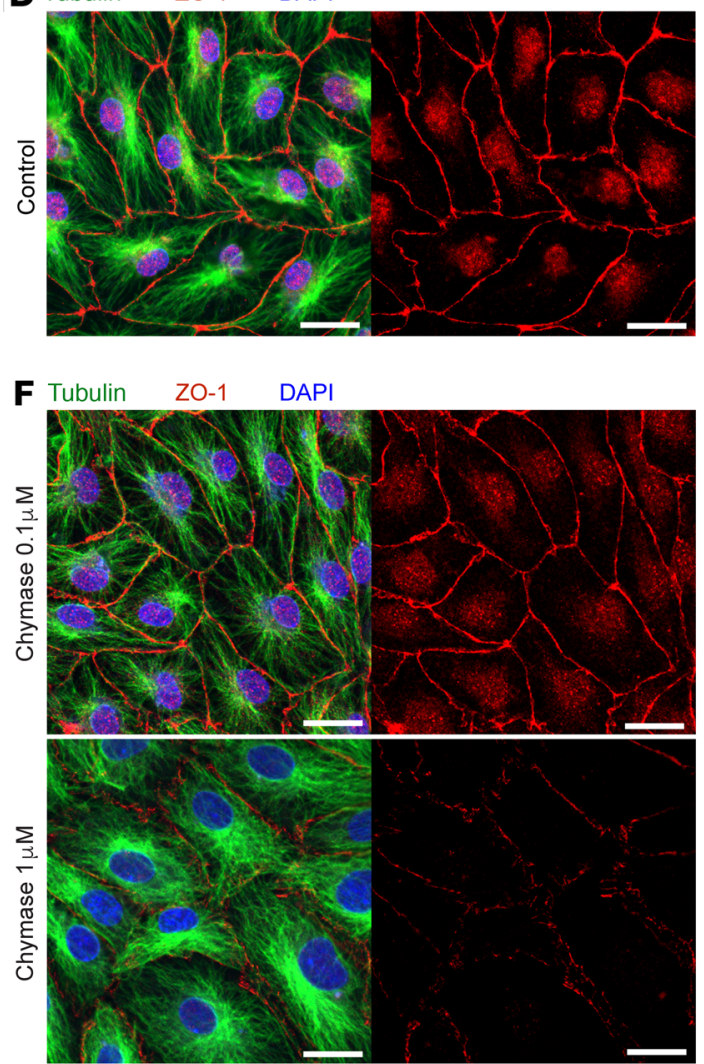

B

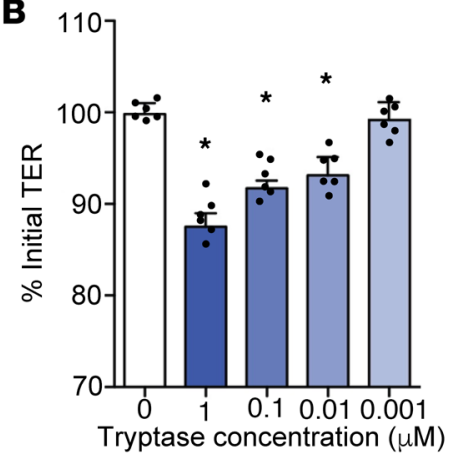

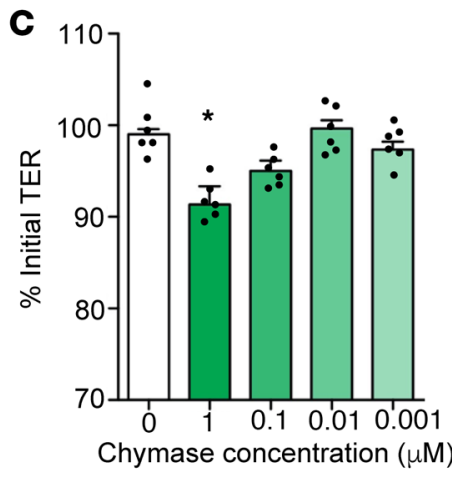
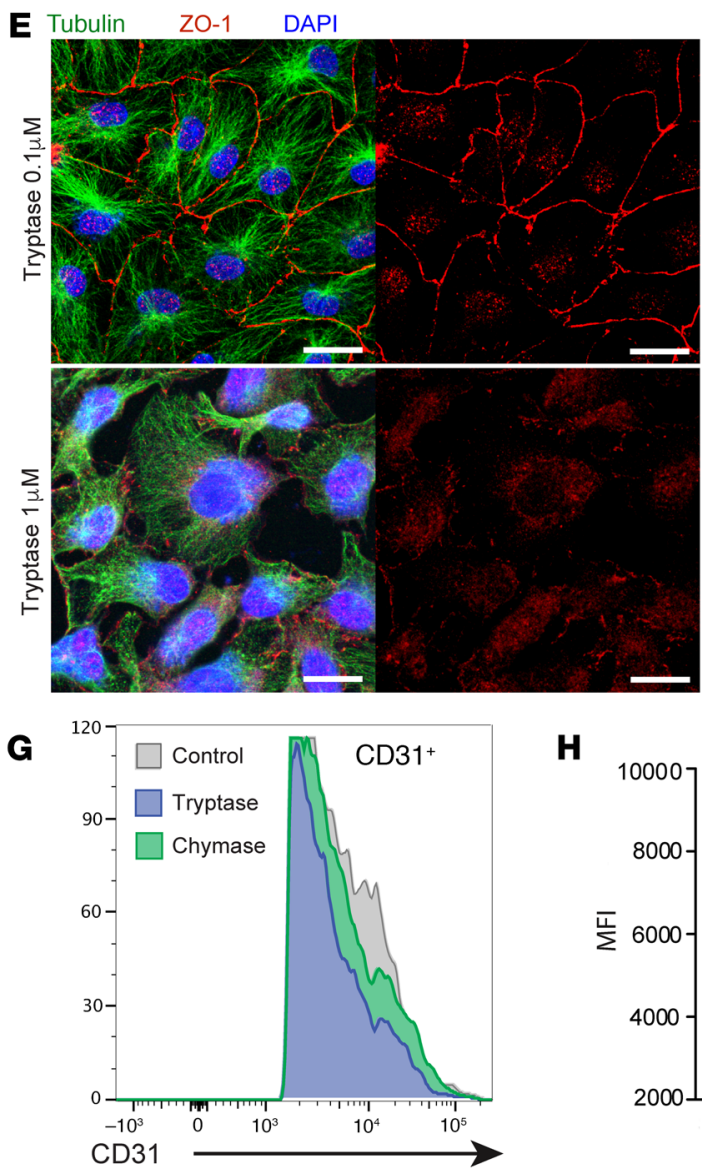

H

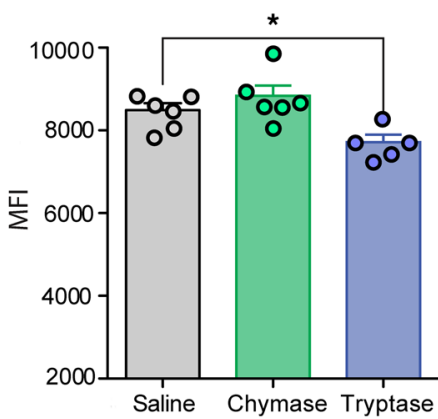

Figure 1. Tryptase and chymase break tight junctions to disrupt endothelial cell contact sites. (A) DENV-stimulated MC supernatant was transferred onto huMEC monolayers or separated into soluble and particulate fractions, followed by transfer onto huMEC monolayers. For controls, DENV-alone or media-alone groups were used. TER of huMEC monolayers was measured 24 hours after treatment. Whole supernatant or isolated MC particles each significantly reduced the TER of huMEC monolayers ( $P<0.05$ by 1-way ANOVA with Dunnett's post test). (B and $\mathbf{C})$ TER of huMEC monolayers after treatment for 24 hours with either purified, recombinant tryptase (B) or chymase (C). ${ }^{*} P<0.05$, decrease in TER over controls by 1-way ANOVA with Dunnett's post test (A-C). (D-F) huMECs were treated with low $(0.1 \mu \mathrm{M})$ or high $(1 \mu \mathrm{M})$ concentrations of either tryptase or chymase for 24 hours, followed by fixation and staining against tubulin (green), nuclei using DAPI (blue), and tight junctions (ZO-1, red). (D) In control cells, tight junctions were intact in between cells, visualized by ZO-1 staining. (E) Tryptase induced a concentration-dependent reduction in Z0-1 staining that appeared disjunctive at low concentrations and absent at high concentrations. Lifting of cells forming gaps was also observed after high-concentration tryptase treatment. $(\mathbf{F})$ Low-concentration chymase had no apparent effect on tight junctions, while staining grew more punctate at high concentrations. For $\mathbf{A}-\mathbf{F}$, data are representative of 3 independent repeats. Scale bars: $25 \mu \mathrm{m}$. (G and $\mathbf{H})$ Levels of CD31 on endothelial cells were measured by flow cytometry on cells isolated from mouse footpads 6 hours after injection of $100 \mathrm{ng}$ of tryptase, chymase, or saline vehicle control. (C) CD31+ cells showed reduced levels of staining after injection of tryptase (representative histogram plots). (H) Comparison of MFI of CD31 staining in mouse footpads ( $n=$ 5-6 each group) showed that tryptase, but not chymase, is sufficient to induce a significant decrease in CD31 staining in vivo (right panel, $P<0.05,1$-ANOVA with Dunnett's post test). For graphs, error bars represent SEM. 

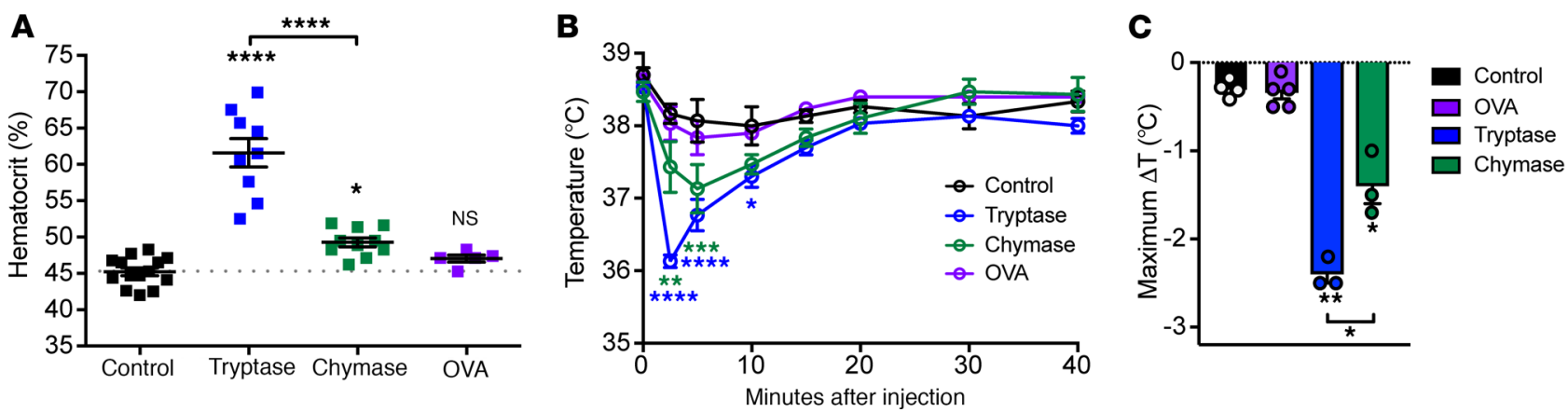

Figure 2. MC proteases promote vascular leakage and shock in vivo. (A) Hematocrit values were obtained 6 hours after injection with saline alone or 30 ng of either tryptase, chymase, or OVA. Means differ significantly by 1-way ANOVA $(P<0.0001)$. Bonferroni's multiple comparison test was used to determine significance among groups. Control, $n=15$; tryptase and chymase, $n=10$; OVA, $n=5$. Data were added from 2 independent experiments. (B and C) Mice ( $n=$ 3-4) were injected with $30 \mathrm{ng}$ each of tryptase, chymase, or OVA i.v., or an equivalent volume of saline was injected for controls. To measure shock, the body temperature of animals was recorded every 5 minutes for the first 15 minutes and subsequently at 10-minute intervals. (B) Both tryptase and chymase caused sudden drops in body temperature, indicative of shock, compared with both OVA and saline control groups. Data were analyzed by 2-way ANOVA with HolmŠidák multiple comparison test to compare temperatures at each time point. (C) The maximal difference in temperature during the time course is presented, suggesting that tryptase treatment causes significantly higher plasma loss in animals compared with chymase, OVA, and saline control groups, determined by 1-way ANOVA with Holm-Šidák multiple comparison test. Data are shown as mean $\pm \mathrm{SEM} .{ }^{*} P<0.05 ;{ }^{* *} P<0.01 ;{ }^{* * *} P<0.001 ;{ }^{* * *} P<0.0001$.

both human tryptase and human chymase, as histidine-tagged recombinant proteins for use in functional assays. The proteins were chromatography purified, tested to determine they were endotoxin free, and tested to verify for functional protease activity. Incubation of tryptase with huMECs resulted in a strong, dose-dependent increase in endothelial permeability, as demonstrated by a drop in the TER (Figure 1B). In contrast, chymase promoted only a moderate decrease in the TER of huMEC monolayers at the highest $(1 \mu \mathrm{M})$ concentration tested (Figure 1C). These data point to the role these proteases could play during DENV disease severity.

Next, we sought to understand the mechanism behind tryptase/chymase-induced endothelial permeability. Since tight junctions between the cells are crucial for maintaining the endothelial barrier function and tryptase, in particular, has been described as cleaving the tight junction component PAR-2 (38), we questioned whether loss of tight junctions would underlie the MC proteaseinduced increases in endothelial permeability. To address this, huMECs were cultured to form a monolayer on glass coverslips, followed by exposing them to 2 different concentrations $(0.1 \mu \mathrm{M}$ and $1 \mu \mathrm{M}$ ) of tryptase or chymase for 24 hours. At 24 hours after treatment, cells were fixed and immunostained for tight junction protein ZO-1 and tubulin to reveal each individual cell cytoskeleton and with DAPI for nuclear localization. The microscopy images show a uniform continuous staining of ZO-1 in untreated control cells (Figure 1D), suggestive of intact endothelial tight junctions. However, treatment of huMEC monolayers with tryptase led to dramatic damage of tight junctions, with ZO-1 staining completely disappearing at the high, $1 \mu \mathrm{M}$ concentration of tryptase (Figure 1E). Individual cells were also observed to lift from the coverslips, leaving large gaps between cells (Figure 1E). This effect was substantial even with low-dose tryptase treatment $(0.1 \mu \mathrm{M})$, where only punctate staining for ZO-1 at the cell borders remained (Figure 1E). Consistent with the TER data presented in Figure 1C, chymase treatment also caused damage to tight junctions, but to a lesser extent compared with that of tryptase treatment (Figure 1F). Collectively, these data show that both tryptase and chymase are able to break tight junctions between endothelial cells, causing increases in endothelial permeability.

Tryptase treatment reduces surface expression of adhesion molecule CD31 on vascular endothelium. To understand in detail how tight junctions are affected in vivo by the MC proteases chymase and tryptase, we measured the surface expression of cell-adhesion molecule CD31 (also called PECAM-1) on vascular endothelial cells after injection of either of these proteases. For this, $100 \mathrm{ng}$ of tryptase or chymase was injected in the mouse rear footpad, followed by harvest of tissue from the footpad after 6 hours. Single-cell suspensions were prepared from the tissue, and endothelial cells were stained using an antibody against CD31, which is both a marker for endothelial cells and a functional component of tight junctions, before being analyzed by flow cytometry. The data demonstrate that the MFI of CD31 staining was significantly decreased upon tryptase treatment compared with that of the saline injection control (Figure $1, \mathrm{G}$ and $\mathrm{H}$ ). Chymase treatment, in contrast, did not influence the surface expression of CD31 (Figure $1 \mathrm{H}$ ). In the context of DENV infection in vivo, $\mathrm{CD} 31$ expression was also found to be significantly reduced on endothelial cells when mice were infected with DENV (Supplemental Figure 1; supplemental material available online with this article; https://doi.org/10.1172/JCI128426DS1). These data suggest that, during DENV infection, tryptase breaks tight junctions between endothelial cells and results in a reduction in the surface expression of the cell-cell adhesion molecule CD31 in vivo. In contrast, the effect of chymase was not significant after injection and did not influence CD31 expression.

MC proteases tryptase and chymase promote vascular leakage and induce hypovolemic shock in vivo. To determine whether MC proteases could have a functional role in plasma loss during DENV infection in vivo, we injected them i.v. in mice, aiming for a final serum concentration of proteins around $10 \mathrm{ng} / \mathrm{mL}$, approximately equivalent to the concentrations of proteases that were previously reported in the serum of human DHF patients (34), assuming an approximately $3 \mathrm{~mL}$ blood volume for a mouse. Mice were injected with chymase or tryptase, and at 6 hours after injection, hemato- 
A

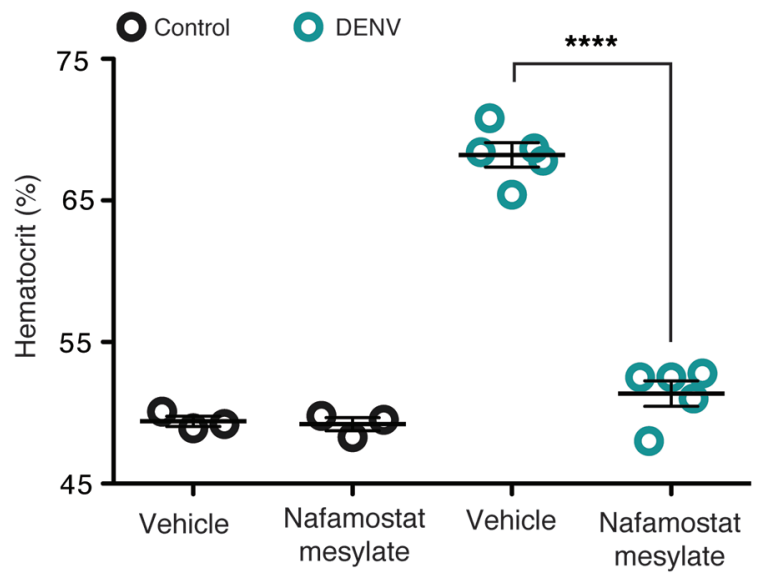

C

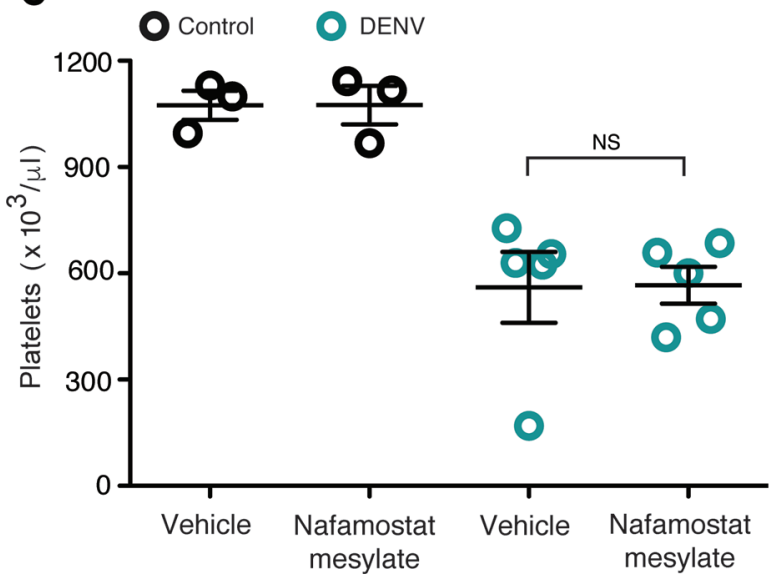

B

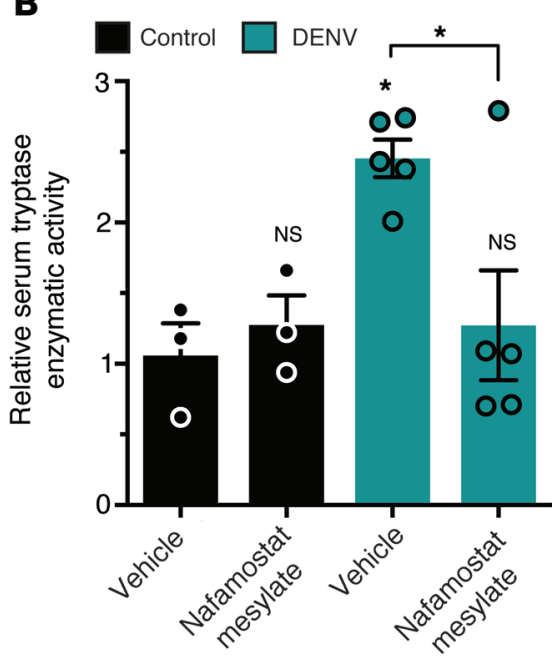

D

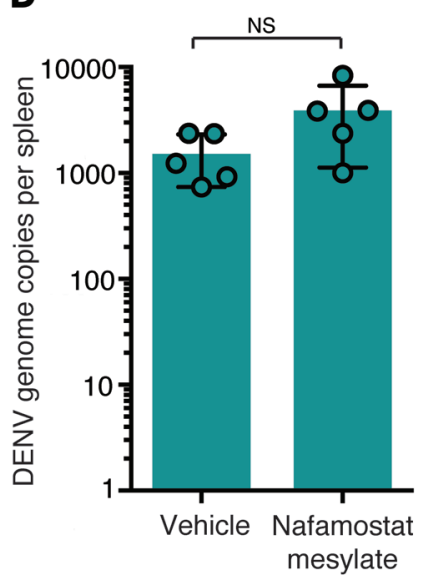

Figure 3. Tryptase inhibition therapeutically blocks vascular leakage during DENV infection. Mice $(n=3-5$ per group) were either mock infected or infected with DENV $\left(1 \times 10^{6} \mathrm{PFU}\right)$, followed by treatment with vehicle control (saline) or using a specific tryptase inhibitor, nafamostat mesylate, at a dose of $0.06 \mathrm{mg} / \mathrm{kg}$. (A) Hematocrit analysis was performed using an automated hematology analyzer on whole blood at 24 hours after treatment. (B) Serum was isolated to measure tryptase activity by enzymatic assay. Only DENV-infected mice that were vehicle treated had elevated tryptase activity over uninfected control group. Nafamostat mesylate treatment reversed tryptase activity to baseline levels. (C) Platelet counts at 24 hours are presented. The data show a strong reduction in DENV-induced vascular leakage upon treatment with tryptase inhibitor, nafamostat mesylate, but no significant difference in platelet counts compared with DENV-infected and vehicle-treated mice. For A-C, statistical significance was determined using 1-way ANOVA with Bonferroni's multiple comparison test. (D) No difference in the DENV burden in the spleen determined by real-time reverse-transcription PCR (RT-PCR) was observed between vehicle- and nafamostat mesylate-treated animals at 72 hours after infection by Student's unpaired $t$ test. For all panels, data are presented as mean \pm SEM. ${ }^{*} P<0.05 ;{ }^{* * *} P<0.0001$.

crit values were measured from the blood to quantify the degree of plasma loss from circulation in mice after treatments. Both chymase and tryptase were sufficient to induce physiologically significant increases in vascular leakage, which was measured as an increase in hematocrit (Figure 2A). However, tryptase resulted in an approximately $16 \%$ increase in the RBC volume in the blood, compared with an approximately $3.8 \%$ increase in $\mathrm{RBC}$ volume after chymase injection (Figure 2A). Injection of a control protein, OVA, did not significantly influence the hematocrit (Figure 2A). To confirm vascular leakage by a secondary method, we injected Evans Blue dye (EBD) 6 hours after injection of either chymase or tryptase and, after an additional 30 minutes, quantified the amounts of dye that leaked into the liver tissue (Supplemental Figure 2). EBD leaked significantly into the livers of mice that were tryptase injected, confirming the induction of vascular leakage, while the increases in EBD in chymase-injected mice were not significant (Supplemental Figure 2). Thus, while both chymase and tryptase can affect vascular homeostasis, tryptase promoted the most substantial vascular leakage.

Next, we questioned whether the rise in hematocrit that occurred during tryptase/chymase treatment could result in hypovolemic shock. The standard way to measure infectious or anaphylactic shock in the mouse model is to record a drop in body temperature (39-41). To test this, mice were given $30 \mathrm{ng}$ of either tryptase, chymase, or OVA i.v., followed by the measurement of temperature at regular intervals. Interestingly, both tryptase and chymase injections resulted in a dramatic drop in the body temperatures of mice, suggestive of a shock (Figure 2B). However, tryptase treatment resulted in a stronger drop in body temperature $\left(-2.5^{\circ} \mathrm{C}\right.$ below normal $)$ compared with chymase treatment $\left(\sim 1.5^{\circ} \mathrm{C}\right)$ and persisted longer (Figure 


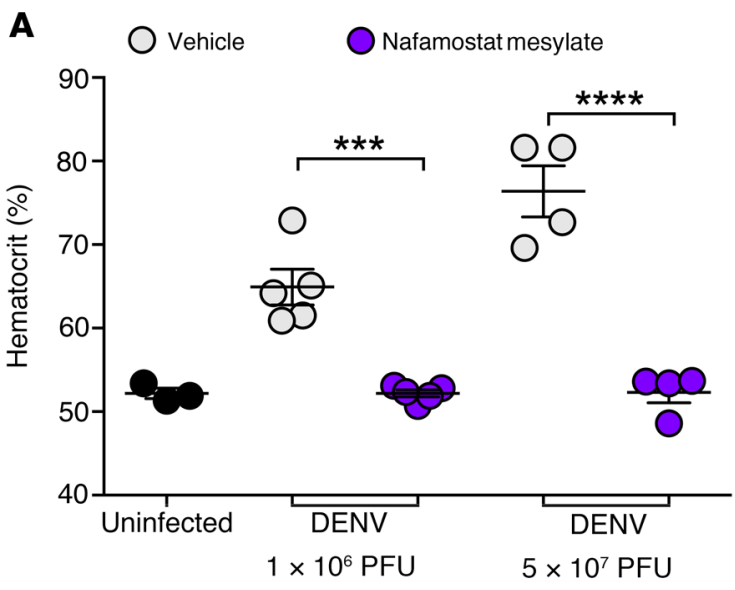

B
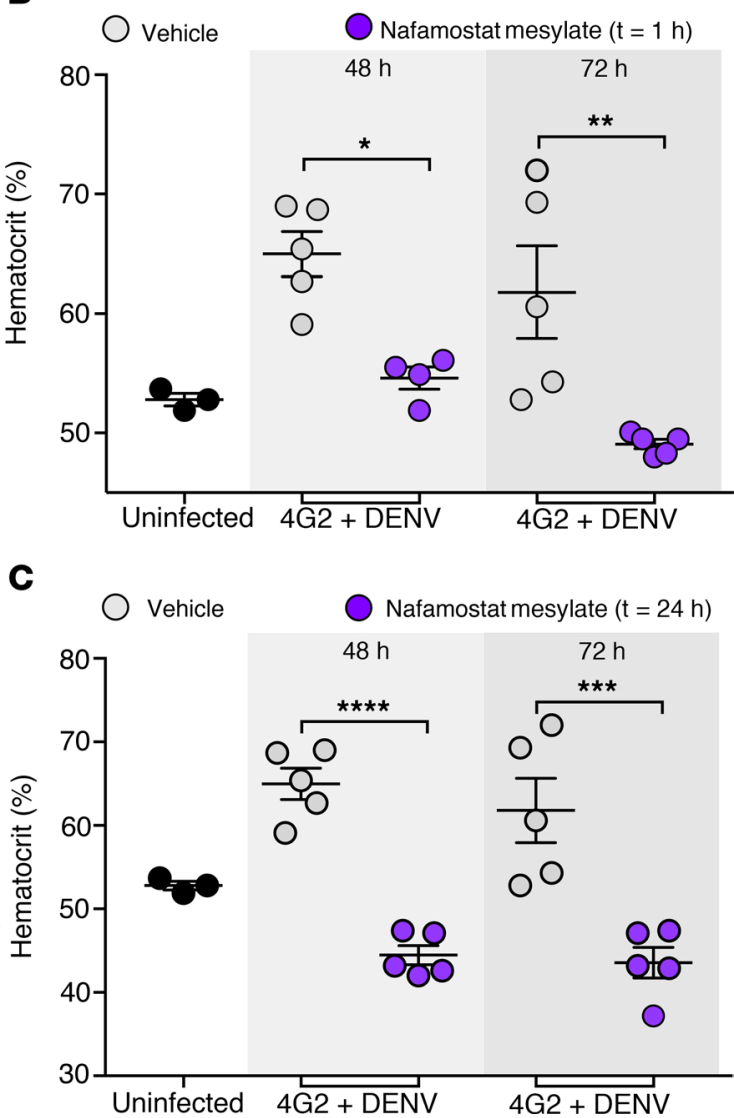

2C). Control animals that were given essentially the same medium in which proteins were diluted did not undergo any shock and only experienced a moderate and brief temperature decline that is likely attributable to the injection of saline, as did animals that were injected with an equivalent amount of the exogenous protein OVA (Figure 2, B and C), which was used as a control for protein injection. Overall, these data show that, although both tryptase and chymase are able to cause plasma leakage in vivo, tryptase has a more profound effect on vascular endothelium with regard to inducing vascular leakage and shock compared with chymase.

Tryptase inhibition protects against DENV-induced hemoconcentration. Based on the observation that tryptase is highly consequential to vascular leakage in DENV-infected animals and suf-
Figure 4. Improvement of vascular leakage in severe DENV infection models. AG129 mice were infected with a low $\left(1 \times 10^{6} \mathrm{PFU}\right)$ or high $(5 \times$ $10^{7} \mathrm{PFU}$ ) dose of DENV and either mock or nafamostat mesylate treated beginning 1 hour after infection. Uninfected, $n=3$; DENV infected, vehicle treated, $n=4-5$; and DENV infected, nafamostat mesylate treated, $n=$ 4-5. (A) At 24 hours after infection, hematocrit was measured. A single treatment of nafamostat mesylate reversed DENV-induced vascular leakage for both infection doses. (B and C) Nafamostat mesylate effectively restored hematocrit values to baseline levels at days 2 and 3 after infection in an antibody-enhanced DENV mouse model. Treatment was initiated (B) 1 hour or delayed (C) 24 hours after infection and given at 24-hour intervals thereafter. Statistical significance was calculated using 1-way ANOVA with Bonferroni's multiple comparison test. ${ }^{*} P<0.05$; ${ }^{* *} P<0.01$; ${ }^{* * *} P<0.001$; ${ }^{* * * *} P<0.0001$. For all panels, data are presented as mean \pm SEM.

ficient to induce shock in vivo, we aimed to ameliorate vascular leakage in DENV-infected animals by therapeutically targeting tryptase. For this, we used the drug nafamostat mesylate, since this drug is a highly specific inhibitor of tryptase at picomolar concentrations and also approved for clinical use for intravascular coagulation $(42,43)$. Animals were treated with nafamostat mesylate after DENV infection, and vascular leakage was measured in the animals by obtaining hematocrit readings. We observed that nafamostat mesylate alone did not influence the hematocrit of healthy uninfected control animals. However, in DENV-infected animals in which strong hemoconcentration indicating vascular leakage was observed during infection, nafamostat mesylate restored the hematocrit to homeostatic levels (Figure 3A). These data are further solidified by measurements of tryptase and chymase activities in the serum of mice from each experimental group. The enzymatic activities of both tryptase and chymase were significantly higher in the DENV-infected but vehicle-treated animals compared with those of the control groups (both mock-infected, vehicle-treated and mock infected, drug-treated) (Figure 3B and Supplemental Figure 3). Treatment of DENV-infected animals with nafamostat mesylate resulted in functional serum tryptase levels that were at baseline, confirming the specific action of this drug in blocking tryptase activity in vivo (Figure 3B). Together, these data demonstrate that tryptase inhibition is able to therapeutically block DENV-induced plasma loss significantly. The specificity of the drug's action on DENV-induced plasma leakage is further shown by the fact that nafamostat mesylate treatment did not alter chymase activity (Supplemental Figure 3) or ameliorate the drop in platelets that is observed during infection (Figure 3C) and did not significantly influence the titers of virus in vivo (Figure 3D). Since tryptase can theoretically influence the coagulation and complement cascades $(44,45)$, we measured levels of complement component C3 in the serum of DENV-infected and vehicleor nafamostat mesylate-treated animals, but observed no changes in the levels of C3 with drug treatment, although C3 was reduced in DENV-infected animals compared with controls (Supplemental Figure 4), suggesting DENV induced a tryptase-independent complement activation. These results support that the therapeutic effects of nafamostat mesylate on DENV-vascular leakage are due to its action as a specific inhibitor of tryptase enzymatic activity.

We repeated our findings in a severe model of DENV viremia to establish whether vascular leakage could also be reversed in those mice. The AG129 mouse line is deficient in receptors for 
A
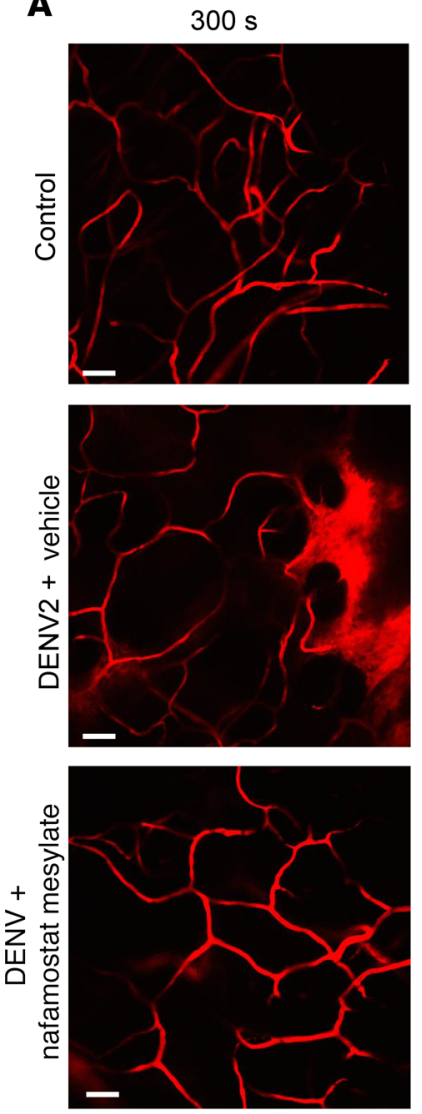

$633 \mathrm{~s}$
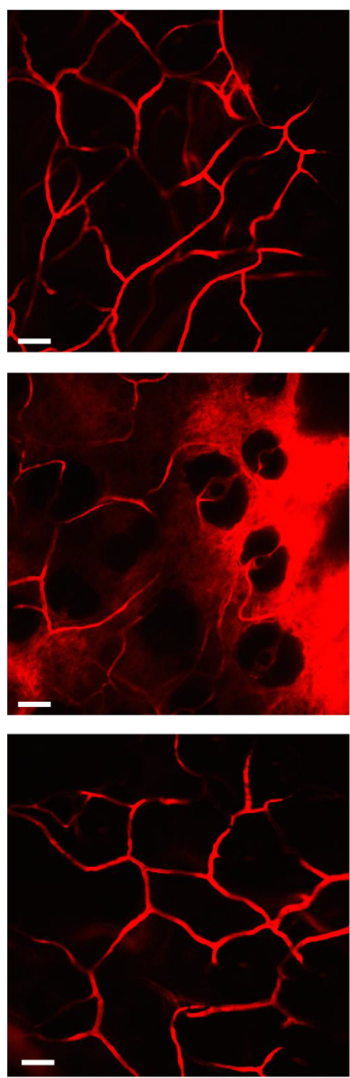

$966 \mathrm{~s}$
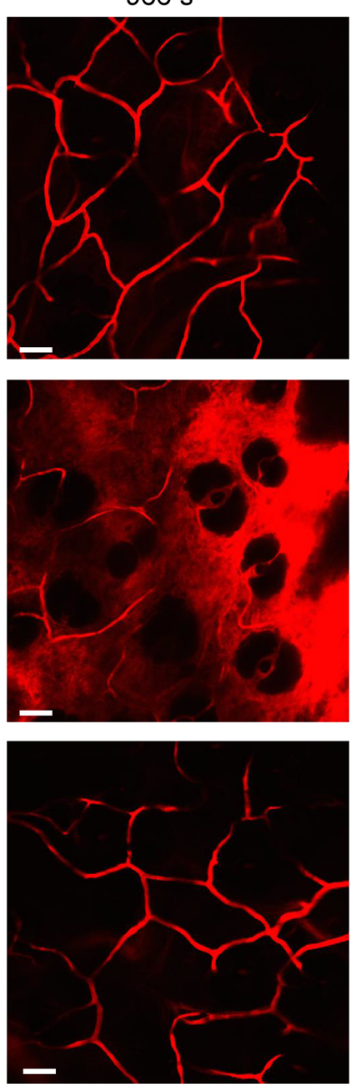

$1298 \mathrm{~s}$
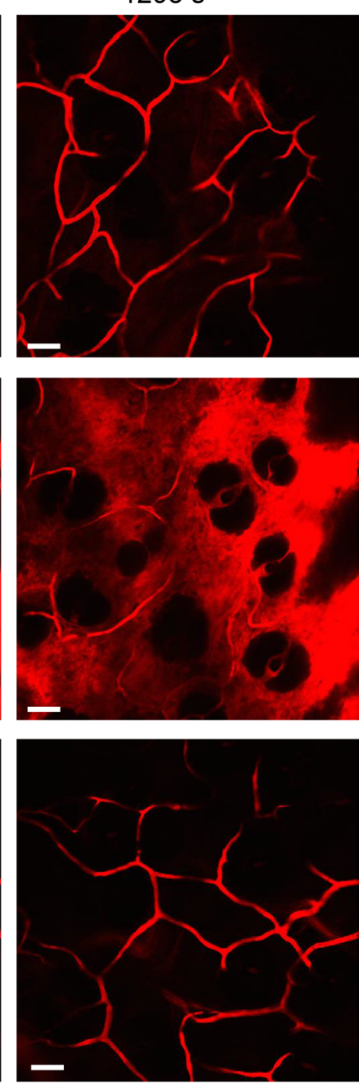

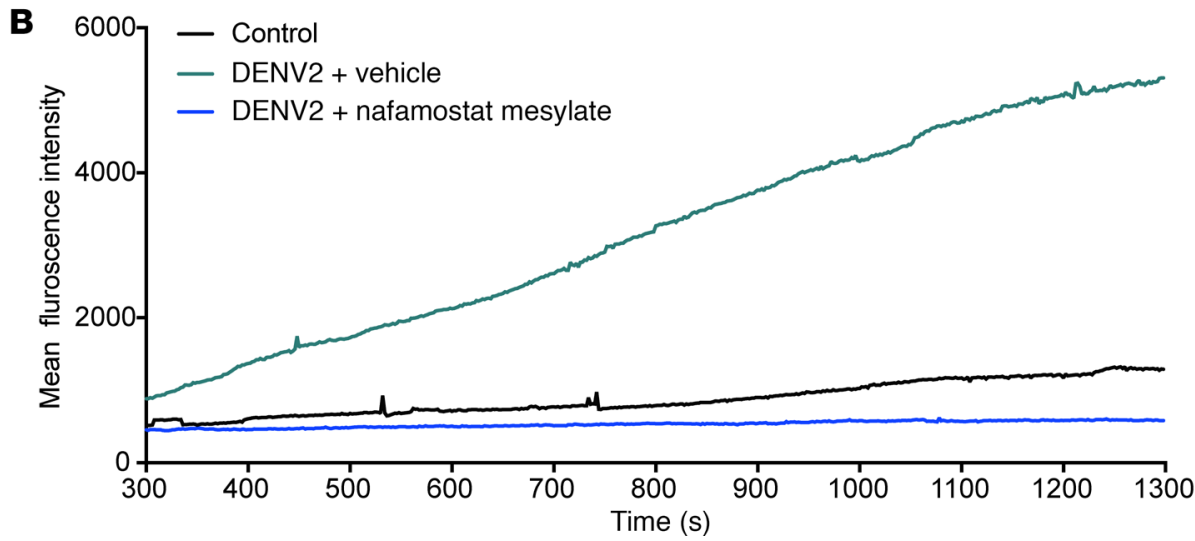

Figure 5. Visualization of inhibition of plasma leakage in DENV-infected mice. AG129 mice were infected with $1 \times 10^{6}$ PFU of DENV i.p. and either treated with nafamostat mesylate or with vehicle. Mice $(n=3), 24$ hours after infection, were injected with $70 \mathrm{kDa}$ FITC-dextran. Two-photon images were acquired continuously at 2-second intervals, beginning 5 minutes after injection, for 18 minutes total. (A) Representative images from the indicated time points after FITC-dextran injection from Supplemental Videos 1-3, showing vascular leakage in the DENV-infected mock-treated ear, while control mice and DENV-infected mice treated with nafamostat mesylate showed no visually discernible vascular leakage. Scale bars: $50 \mu \mathrm{m}$ (B) MFI in the acquired images over time is presented. Intensity was measured by averaging 10 areas in the interstitial space. Data are representative of 3 independent experiments.

both type I and type II interferons and has been used extensively to study DENV infection in vivo. Nafamostat mesylate was effective in reducing hematocrit values in AG129 mice given both high and low inoculating doses of DENV (Figure 4A). Next, we evaluated whether tryptase inhibition can also be effective in a severe model of dengue disease, characterized by antibody-dependent enhanced infection (ADE) of DENV in AG129 mice. Mice were given an enhancing concentration of antibody $4 \mathrm{G} 2$, followed by DENV infection, which, as expected, led to increased virus titers through ADE (Supplemental Figure 5). Subsequent to infection, mice were treated daily with the tryptase inhibitor nafamostat mesylate. Nafamostat mesylate treatment significantly reduced the hematocrit values on days 2 and 3 after infection (Figure $4 \mathrm{~B})$, demonstrating that the tryptase-dependent mechanism for reducing vascular leakage is consistent in this second model that involves antibody-enhanced disease. We also measured the serum tryptase and chymase enzymatic activity in serum from each experimental group. Consistent with our data in WT mice 
A

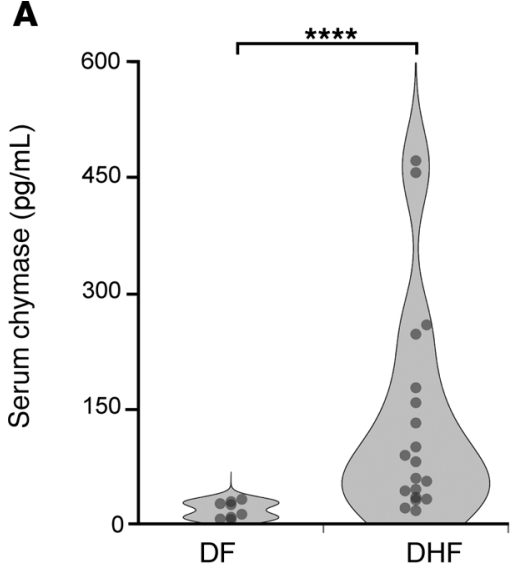

B

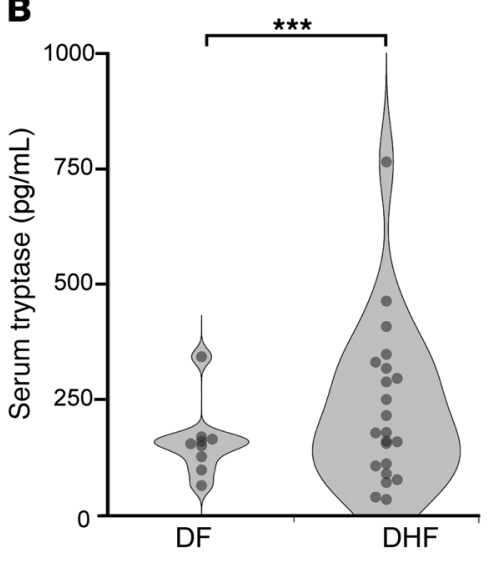

C

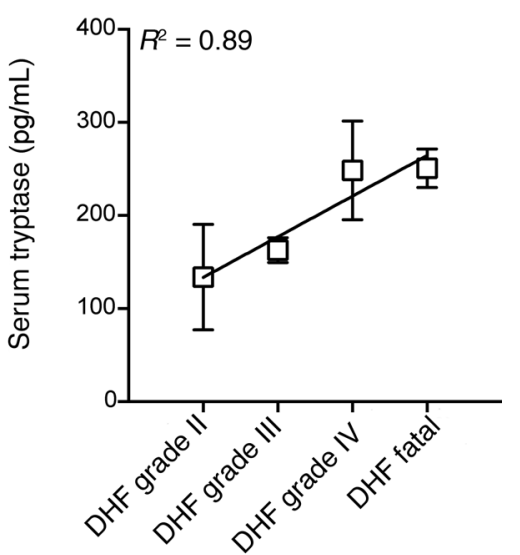

D

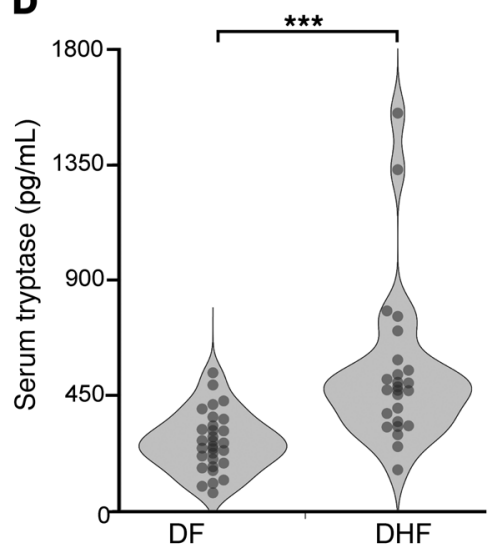

E

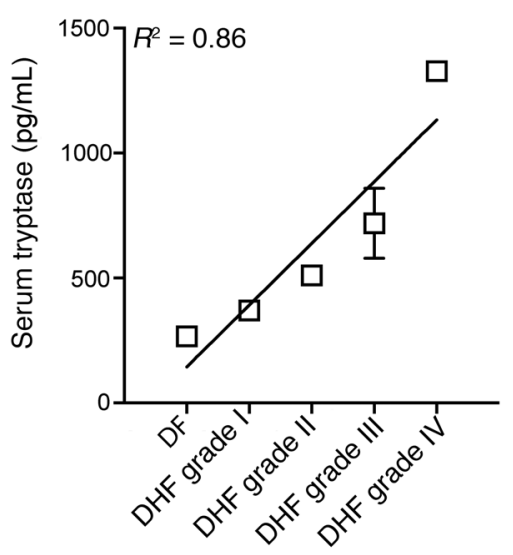

Figure 6. Serum tryptase levels are correlated with DHF/DSS in humans. Serum samples from virologically confirmed hospitalized dengue patients in Jakarta, Indonesia, in 1975-1978 ( $n=9 \mathrm{DF} ; n=25 \mathrm{DHF}$ ) were tested retrospectively in a blinded manner for (A) chymase and (B) tryptase. Both were significantly increased $(P<0.0001$ and $P=0.0004$, respectively, determined by Student's unpaired $t$ test) in patients with DHF compared with DF. (C) Mean serum tryptase levels for each grade of DHF were also strongly correlated with the grade of DHF based on the patient's reported symptoms. $P=0.05 ; R^{2}=$ 0.89. ( $\mathbf{D}$ and $\mathbf{E}$ ) Tryptase was measured in a second cohort of prospectively obtained patient samples from virologically confirmed dengue patients $(n=30$ DF; $n=25$ DHF) in Sri Lanka, Colombo, in 2012-2013. (D) Serum tryptase levels were significantly elevated in serum samples from DHF versus DF patients $(P=0.0005)$. (E) Mean serum tryptase levels in Sri Lankan samples were strongly correlated with disease severity. $P=0.02 ; R^{2}=0.86$. For all panels, data are presented as mean \pm SEM.

(Figure 3B and Supplemental Figure 3), we observed that nafamostat mesylate treatment significantly reduced tryptase activity in AG129 mice on days 2 and 3 after infection (Supplemental Figure $6 \mathrm{~A}$ ), yet had no effect on serum chymase activity (Supplemental Figure 6B), again confirming the specificity of this tryptase inhibitor. Finally, we examined the efficacy of delayed treatment, in which AG129 mice infected with the same antibody-enhanced strategy were treated with nafamostat mesylate daily, but beginning 24 hours after infection (Figure 4C). Even with delayed treatment, nafamostat mesylate had a significant therapeutic effect and reduced hematocrit levels following infection (Figure 4C).

To confirm the influence of nafamostat mesylate on vascular leakage by an alternate method, we performed intravital multiphoton microscopy. The ears of AG129 mice that had been mock infected (Supplemental Video 1), infected with DENV and given vehicle control injections (Supplemental Video 2), or infected with DENV and given injections of nafamostat mesylate (Supplemental Video 3) were imaged after injection of FITC-dextran dye, which was used to visualize vascular leakage. Still images from various time points beginning 24 hours after infection from Supplemental Videos 1-3 are presented in Figure 5A. While dye was confined to the blood vessels of control uninfected tissues for the duration of imaging, it could be visualized leaking into the tissue in DENV-infected animals (Figure 5A and Supplemental Videos 1 and 2). Nafamostat mesylate treatment reversed the leakiness of the vasculature in DENV-infected animals (Figure 5A and Supplemental Video 3). Quantification of the dye leakage in the videos showed that DENV infection allowed dramatic increases in dye detection over the course of visualization, while only a slight increase occurred in control uninfected tissues (Figure 5B). In contrast, fluorescence detection remained near baseline in DENVinfected but nafamostat mesylate-treated animals (Figure 5B). Additional experiments at 48 hours after infection for the same groups as described above confirmed the efficacy of nafamostat mesylate in blocking DENV vascular leakage (Supplemental Videos 4-6). These results further support tryptase as a mechanism of DENV-induced vascular leakage and also suggest this enzyme may be an effective therapeutic target. 
Tryptase levels correlate with DENV severity in humans. After experimentally establishing that both tryptase and chymase could negatively influence the integrity of the vascular endothelium during DENV infection and that they are sufficient to induce plasma leakage in mice, we did a retrospective study of acute human serum samples collected during an epidemic that occurred in Jakarta, Indonesia, in 1975-1978 (46-48). The 34 samples from virologically confirmed DENV-infected patients clinically graded to have a range of DENV pathologies from DF to fatal DHF (Figure 6A) and representing multiple serotypes (18\% DENV-1, 24\% DENV-3, 3\% DENV-4, and 55\% serotype undetermined) were viewed in a blinded manner as to clinical severity of the patient and virus serotype. Consistent with our earlier published data (34), levels of chymase were significantly elevated in serum of patients with severe DENV outcomes, consistent with the clinical definition of DHF (Figure 6A). Remarkably, the levels of tryptase were also significantly elevated during DHF (Figure 6B). However, chymase levels showed an approximately $800 \%$ increase in DHF patients over DF patients versus a 75\% increase in tryptase for DHF patients over DF patients (Figure 6, A and B). These findings are consistent with our previous report that chymase is a robust biomarker for DHF (34). Next, we assessed whether there was a correlation between the levels of tryptase or chymase and the severity of DHF (DHF grades II-IV and fatal DHF). DHF was graded according to WHO guidelines (3). Interestingly, levels of tryptase followed a strong linear correlation with the severity of DHF in human patients (Figure 6C). However, although the levels of chymase were higher in DHF patients, there was no statistically significant correlation observed with the severity of DHF $\left(R^{2}=0.5, P=0.3\right.$; Supplemental Figure 7A). These data show that increased levels of serum tryptase were correlated with increased disease severity in patients (Figure 6, B and C). To confirm this finding, a more recent cohort of serum samples prospectively collected in Sri Lanka in 2012-2013 were tested. These samples were previously shown to have significantly elevated chymase levels in DHF patients compared with DF patients (36). Consistent with findings in the Indonesian cohort, DHF patients compared with DF patients had significantly higher levels of serum tryptase (Figure 6D). Furthermore, tryptase levels (Figure 6E), but not chymase levels (Supplemental Figure 7B), were significantly correlated with the grade of DHF. Collectively, these data suggest that tryptase is a mechanistic correlate of vascular leakage during severe dengue that could also potentially be used as a prognostic marker for DHF severity.

\section{Discussion}

Multiple theories have been put forward to explain how DENV vascular leakage syndrome is initiated and sustained in vivo. In the absence of strong and consistent evidence that endothelial cells are directly infected by DENV in humans, immunemediated pathology is presumed to underlie the mechanism of increased endothelial permeability during DHF and DSS (2). In this work, we describe a mechanism of vascular leakage during DENV disease and show that the MC-derived protease tryptase is a host protein that is consequential for breaking endothelial tight junctions. Tryptase is a serene protease that functions in vivo to cleave a list of targets including kininogen, complement factor C3, clotting factor XII, fibrinogen, and others (49). Tryptase also regulates the activity of other serine proteases, such as prourokinase, an important coagulation factor (50). Interestingly, an early report describing fatal DHF in the Philippines reported low levels of circulating fibrinogen accompanying intravascular coagulation (51), which would be consistent with degradation of this product by tryptase. Decreased serum fibrinogen, high levels of fibrinogen split products, and intravascular coagulation were also observed subsequently in a cohort of patients from Thailand (52), although it should be noted that intravascular coagulation is a rare outcome of dengue (52). Indeed, in our study, injection of tryptase alone was sufficient to induce shock in mice. A high-affinity tryptase-specific inhibitor, nafamostat mesylate, limited DENV-induced vascular leakage in vivo. This drug is currently approved for use to treat disseminated intravascular coagulation (42), which is also a potential clinical sign accompanying DSS (52-54). It is also effective for treatment of conditions in which limiting endothelial activation and/or limiting coagulation is beneficial, such as renal ischemia reperfusion injury $(55,56)$. While nafamostat mesylate can inhibit some other serine proteases at high concentrations, it has been shown to be highly specific for tryptase at the concentrations used in this study (57-59). Some additional pathways that can be influenced directly or indirectly by tryptase include PARs and the fibrinolytic systems $(38,44$, 50), as previously discussed, as well as the kallikrien-kinin system and complement activation pathways $(45,60)$. Tryptase is also a known glycocalyx sheddase (61), and glycocalyx degradation has been observed to occur during human dengue infection $(62,63)$. Although not tested here, the tryptase-dependent effect of nafamostat mesylate on coagulation factors such as fibrinogen (64, 65) could potentially be an added advantage for the treatment of severe DENV disease, since fibrin split products and intravascular coagulation are known to occur during DHF/DSS.

Importantly, nafamostat mesylate was effective in multiple models of DENV infection, including immunocompetent and immunocompromised mice and antibody-enhanced models of severe DENV disease. We also observed efficacy with delayed treatment. Antibody enhancement is thought to promote severe disease due to antibody-dependent uptake of virus particles by $\mathrm{Fc}$ receptor-bearing cells, and we have recently shown that FcRdependent enhancement of MC degranulation occurs as a second antibody-dependent mechanism leading to enhanced vascular leakage (35). In humans, biomarkers of MC activation, such as chymase, are detected at higher levels in patients with secondary infection, supporting the idea that enhanced MC degranulation occurs during secondary DENV infection (34), which could be a factor in the efficacy of nafamostat mesylate in the animal model of antibody-enhanced severe disease, in addition to the possibility of increased activation of MCs due to higher titer of virus resulting from traditional ADE. Aside from antibody-enhanced degranulation, traditional ADE can allow MCs to become infected, which could augment production of transcriptionally activated inflammatory mediators, such as cytokines, further during secondary infection (66). In human serum samples examined from two independent cohorts of patients with severe disease, we show that the levels of tryptase were correlated with the grades of DHF in DENV patients. Our results emphasize the role of tryptase in DENV- 
induced vascular permeability and raise the possibility of it being a therapeutic target in treating severe dengue patients at risk for hemorrhage and shock.

We have previously demonstrated that MCs induce vascular leakage during DENV infection in animal models $(34,35)$. However, the mechanisms of action of MC products on the vasculature and the mediators involved in vascular permeability in this context were previously unknown. MCs can release a multitude of soluble mediators (lipids, cytokines, chemokines, and growth factors) as well as particulate mediators in the form of exocytosed granules. These granule structures are known to contain many vasoactive and immune-modulatory products, such as proteases, histamine, heparin, TNF, antimicrobial peptides, and others (23). To begin to address which of these many mediators contributes most substantially to DENV vascular leakage, we fractionated the products released by DENV-activated MCs into soluble and particulate fractions and applied them to endothelial monolayers. Serum from DENV patients is also sufficient to induce breakdown of tight junctions of endothelial monolayers ex vivo (67). Our TER measurements of these huMEC monolayers revealed that the MC granule-associated products, and not the soluble mediators, disrupted endothelial monolayer integrity severely. The dominant MC proteases, tryptase and chymase, constitute the majority of the proteins that are contained within granules. Expression of tryptase and chymase is unique to MCs, and their roles in viral pathogenesis are unknown. Chymase is best recognized as an angiotensin-converting enzyme, but it also can influence the vasculature in several ways. For example, chymase is thought to degrade some extracellular matrix components and has been shown to cleave endothelin-1, promoting vasoconstriction (68). We also recently showed that chymase is important for inducing permeability at the blood brain barrier during Japanese encephalitis virus (JEV) infection (69). Tryptase is known to have a more profound and direct influence on peripheral vascular permeability by cleaving PARs at the interendothelial junctions (38). Using recombinant purified chymase and tryptase, we show here that exposure of endothelial monolayers to tryptase breaks endothelial tight junctions in a dose-dependent manner. This was further verified by injecting tryptase in vivo, which was sufficient to reduce expression of the adhesion molecule CD31 on vascular endothelium and to induce vascular leakage and shock in mice. Although to a lesser extent at the concentration given, chymase was also able to induce moderately increased hematocrit levels that were not accompanied by significantly increased leakage of EBD into tissues at the time point assessed. The influence of chymase on hematocrit levels here could potentially occur through its function of degrading the extracellular matrix (70). The effects of chymase on endothelium could be compounded by the presence of TNF, which is also contained within MC granules (30) and, furthermore, thought to be a possible contributing factor to DENV-induced immune pathology (6).

Notwithstanding these observations, there are other factors that are thought to contribute to vascular permeability during DENV infection. One such host molecule already discussed is TNF, which is not only prestored within MC granules, but also produced abundantly by cells that are infected by DENV. Some studies have shown that TNF blockade can limit vascular pathology and promote survival of DENV-infected animals (6). However, most stud- ies implicating TNF experimentally were performed in mice that were immunocompromised and lacking the IFN-response system (6). This experimental system has the potential to overemphasize the virus replication-dependent aspects of vascular permeability and may result in replication of virus within cell types that would be resistant to infection in IFN-competent animals (2). Recent studies have shown that A129 mice, which are deficient in receptors for type-I interferons, are susceptible to maternal antibody-enhanced lethal DENV infection that is TNF dependent, but do not show significantly increased levels of vascular leakage (71). Moreover, in human studies, TNF could not be consistently linked to DENV severity $(2,72)$. Recently, DENV NS1 protein has also been implicated in vascular leakage in IFN-deficient mouse models (13). Although NS1 may contribute to vascular permeability, further studies are needed to reconcile the observations of some studies that NS1 levels cannot be correlated with severe disease (14-18), including further attempts to control for the potential of antibodies to influence the kinetics of host clearance of NS1 and/or detection of the NS1 protein in the serum. MC degranulation in response to DENV is also dose dependent; thus, higher levels of DENV should also promote heightened degranulation responses, but the reactivity of individual patients to unique amounts of viral antigen could still vary. Identification of MCs as a potential source of vasoactive factors also opens the possibility of additional host-associated factors that influence $\mathrm{MC}$ function that might also then influence DENV disease severity, such as the patient's allergic state, commensal microflora (including parasitic infections), exposure to allergy medications, homeostatic mechanisms for protease inhibition, and genetic factors governing $\mathrm{MC}$ activation and distribution in vivo.

Since MC proteases are presynthesized and some of these enzymes are stored within MCs exclusively, this might also explain why many studies that have attempted to interrogate the pathways involved in DENV-induced vascular leakage through transcriptional profiling or by examining the responses of various infected cell types have not identified increased tryptase or chymase as associated with DENV infection. We have previously reported that the MC protease chymase is a biomarker of disease severity and present in the serum of DHF patients at levels of approximately 10-fold those of DF patients (34). Consistent results were observed in this study, examining cohorts of patients from Indonesia and Sri Lanka. In this case, both chymase and tryptase were elevated in the serum of DHF patients. While chymase may be a more precise biomarker of MC activation during DENV infection, our data show that tryptase is mechanistically more consequential for leakage and shock. We think that this difference could be partially attributed to the difference in functional stability of these proteins in the circulation. The functional halflife of tryptase in the plasma is approximately 8 minutes, which is further stabilized in the presence of heparin for approximately 2 hours (73). Chymase, in contrast, is very sensitive to the presence of protease inhibitors in the plasma, such as secretory leukocyte proteinase inhibitor (SLPI) (74), which could reduce chymase activity in spite of having high circulating protein levels. Furthermore, in contrast to tryptase, SLPI activity is greatly enhanced in the presence of heparin, which was shown to limit the functional half-life of chymase to 0.5 seconds (74). This is consistent with the observation that injection of high levels of tryptase led to a more 
prolonged drop in temperature in animals, while an equivalent concentration of chymase resulted in only a transient temperature drop. We believe that, even though both tryptase and chymase are fairly stable proteins structurally, the differences in their functional enzymatic stability could explain why tryptase is a more potent inducer of endothelial dysfunction during severe DENV disease. Since human MCs display heterogeneity based on their tryptase and chymase content, the types of MCs that are activated might play a role in the severity of disease. While all mature human MCs are thought to contain tryptase within their granules, some MCs have nearly undetectable levels of chymase (75).

Our results also do not suggest that MC proteases must act alone in their ability to induce vascular leakage during DENV infection. We believe that other factors that are produced both by MCs and also by independent lineages of cells (particularly those experiencing viral replication) are likely to contribute to vascular leakage. It is important to note that patients with mild DENV infections display signs of microvascular permeability, such as bruising, purpura, and edema (2). This is also the case during the acute phase of disease for those who subsequently develop severe complications. Another unknown is the role of the strain of DENV in activating MCs. It is known that different strains may have variable infectivity for target cells, which may influence transmission dynamics and virulence. Nevertheless, MCs may be key for initiating the substantial vascular leakage that is characterized by severe DENV infection through their ability to degranulate in response to DENV and the ability of their product, tryptase, a dominant MC granule component, to cleave PARs at endothelial junctions and to induce shock.

\section{Methods}

Cell lines, virus strains, and culture conditions. The huMEC primary cell line HMVEC-d Ad-Dermal MV Endo Cells (Lonza, CC2543) was maintained in EGM-2MV BulletKit media (Lonza, CC3202). The human clinical isolate of DENV2, strain Eden2, which was obtained from the Early Dengue Infection and Outcomes Study (Eden) (76), was maintained at low passage as previously described $(24,34)$.

Measurement of endothelial activation and permeability. huMECs were grown in $3 \mu \mathrm{m}$ inserts (BD Biosciences) inside 24-well plates for 4 5 days to form a monolayer. huMECS were then incubated for 24 hours with different concentrations of either tryptase or chymase $(1 \mu \mathrm{M}, 0.1 \mu \mathrm{M}, 0.01 \mu \mathrm{M}$, or $0.001 \mu \mathrm{M})$. Monolayer permeability was measured by acquiring TER readings at baseline $(t=0)$ and 24 hours after treatment with tryptase, chymase, MC supernatants, or appropriate controls. Human ROSA MCs $\left(3 \times 10^{6}\right.$ cells) $(77)$, a gift from Michel Arock (Ecole Normale Supérieure de Cachan, Cachan, France), were treated for 1 hour with either DENV (MOI1) or control media (untreated), after which the cellular fraction was removed by 2 rounds of centrifugation at $500 \mathrm{~g}$ for 5 minutes. The particles were then pelleted from whole supernatant by spinning at $12,000 \mathrm{~g}$ for 10 minutes at $4^{\circ} \mathrm{C}$. Soluble fractions were collected, and particles were washed and resuspended in maintenance media, followed by exposing them to huMECs monolayers. TER readings were obtained using the Millipore Millicell-ERS (Electrical Resistance System).

Immunofluorescence assay. huMECs were grown on coverslips (Warner Instruments) inside 24-well plates for 2-4 days to form a monolayer. huMECS were then incubated with various concentrations of tryptase or chymase $(1 \mu \mathrm{M}$ or $0.1 \mu \mathrm{M})$. Treated huMECs were incubated for 24 hours, followed by fixation with paraformaldehyde. Coverslips were then washed with PBS and blocked using 0.1\% saponin in 1\% BSA in PBS (permeabilizing buffer). Primary antibodies against $\alpha$-tubulin (GeneTex, catalog GTX11302) and ZO-1 (Invitrogen, catalog 402200) were added to permeabilization buffer and incubated overnight, followed by washing using permeabilizing buffer. Next, the secondary antibodies, anti-mouse-conjugated FITC (Jackson ImmunoResearch, catalog 115-096-006) and anti-rabbit-conjugated Alexa Flour 660 (Thermo Fisher Scientific, catalog A21073) were added in permeabilizing buffer and incubated for 2-4 hours. Finally, coverslips were mounted using ProLong Gold Antifade reagent containing DAPI (Invitrogen, catalog P36931). Cell images were obtained using the LSM710 Carl Zeiss Confocal Microscope with a $\times 63$ objective lens.

Cloning and purification of MC proteases. RNA was isolated from huMCs using the RNeasy Mini Kit (QIAGEN), and cDNA was made using the cDNA Synthesis Kit (Bio-Rad). For chymase, PCR amplification was performed with the following primers: 5 '-CGGCTCATATGATGCTGCTTCTTCCTCTC-3' and 5'-ATACTCTCGAGTTAATTTGCCTGCAGGATCTG- $3^{\prime}$, in which the underlined sequences correspond with Nde I and Xho I restriction sites. For tryptase, PCR amplification was performed with the following primers: $5^{\prime}$-AGTCTCATATGATGCTGAGCCTGCTGCTGCTGGCG-3' and 5'-CAATGAAGCTTTCACGGCTTTTTGGGGACTAGTGGT-3', in which the underlined sequences correspond with Nde I and Hind III restriction sites. PCR products were subsequently cloned in the pET28a Vector (Novagen) using the restriction sites included within the amplification primers. The recombinant pET28a vectors containing either human tryptase or chymase were sequenced to verify in-frame insertion. Recombinant pET28a plasmids were then transformed into E. coli BL-21 (DE3) cells, which were grown in Luria-Bertani (LB) broth containing kanamycin $(50 \mu \mathrm{g} / \mathrm{ml})$ at $37^{\circ} \mathrm{C}$ until an OD-600 of 0.4-0.6 was reached. Protein expression was then induced with $1 \mathrm{mM}$ IPTG overnight at $16^{\circ} \mathrm{C}$. Cell lysates were sonicated, and clarified supernatant was loaded into HisTrap columns (GE Healthcare). HPLC purification was performed according to the manufacturer's instructions under hybrid conditions using an AKTA machine (GE Healthcare). Recombinant active human chymase (CSBYP005599HU) and tryptase (CSB-YP024128HU) expressed in yeast were purchased from Cusabio. Catalytic active sites are highly conserved among human and mouse tryptase and chymase, and, therefore, these proteins are functionally active in vivo (78).

Mouse experiments. WT mice on a C57BL/6 background were purchased from InVivos, and AG129 mice, originally purchased from B\&K Universal, were a gift from Sylvie Alonso (National University of Singapore). For all strains, 6- to 8-week-old female mice were used for experiments. For in vivo studies, due to the large amounts of protein required, yeast-produced MC proteases were purchased from Cusabio, and OVA was purchased from MilliporeSigma. Mice were administered chymase, tryptase, or OVA in PBS via tail-vein injection at the concentrations provided in the figure legends. Blood was collected 6 hours after injection, and hematocrit values were acquired using an AcT Diff Automated Hematology Analyzer (Beckman Coulter). For experiments involving flow cytometry to assess endothelial cells ex vivo, $100 \mathrm{ng}$ of chymase or tryptase or $1 \times 10^{6} \mathrm{PFU}$ of DENV was given by subcutaneous injection in the mouse footpads. Shock was measured quantitatively in mice by recording the temperature at regular intervals using a rectal probe after injection of $30 \mathrm{ng}$ of chymase or 
tryptase by tail vein. For drug studies, nafamostat mesylate (MilliporeSigma, N0289) was given to mice $(0.06$ or $0.6 \mathrm{mg} / \mathrm{kg}$ ) daily i.p. in a $50 \mu \mathrm{l}$ volume of saline, and saline alone served as the vehicle for controls, beginning 1 hour or 24 hours after infection, as indicated in figure legends. Hematocrit and platelet readings were obtained from whole blood collected via cheek vein, using an automatic hematology machine at indicated time points of 24 hours, 48 hours, or 72 hours. Virus quantification was performed after RNA isolation from the spleen at 24 hours, 48 hours, or 72 hours after infection. cDNA was synthesized from $1 \mu \mathrm{g}$ of RNA using the iScript cDNA Synthesis Kit (Bio-Rad) with the addition of primer 5'-TTGCACCAACAGTCAATGTCTTCAGGTTC-3' to synthesize viral RNA to cDNA. Real-time PCR was performed using SYBR Green reagent (Bio-Rad), and the following DENV2 primers were used: forward, 5'-TCAATATGCTGAAACGCGCGAGAAACCG -3'; reverse, 5'-CGCCACAAGGGCCATGAACAG-3'. Systemic infection in AG129 mice was achieved by injecting $1 \times 10^{6}$ or $5 \times 10^{7} \mathrm{PFU}$ of DENV via the i.p. route. For the severe DENV infection model of antibody-dependent enhancement, AG129 mice were passively given $50 \mu \mathrm{g} /$ mouse of antibody $4 \mathrm{G} 2$, followed by infection with a high dose $\left(1 \times 10^{8} \mathrm{PFU}\right)$ of DENV i.p. after 24 hours, according to a published protocol (79). Mice were treated with $0.6 \mathrm{mg} / \mathrm{kg}$ of nafamostat mesylate at 24-hour intervals.

Intravital microscopy. AG129 mice were infected with $1 \times 10^{6} \mathrm{PFU}$ DENV by i.p. injection. Mice were either mock treated with vehicle or treated with nafamostat mesylate $(0.6 \mathrm{mg} / \mathrm{kg}) 1$ hour prior to infection or 24 hours after infection. Mice were anesthetized and injected with $100 \mu \mathrm{l}$ of $50 \mathrm{mg} / \mathrm{ml} 70 \mathrm{kDa}$ FITC-Dextran (MilliporeSigma, 46945) (80), and 2-photon images of the mouse ears were acquired using a Bergamo II Series Multiphoton Microscope from Thorlabs 5 minutes after FITC-dextran injection every 2 seconds for 18 minutes $(920 \mathrm{~nm}$ laser), as described previously (81). For quantifying vascular leakage, 10 areas of equal size were marked near blood vessels and the fluorescence intensity was measured using FIJI (82). MFI for each time point was plotted using GraphPad Prism. Images were converted to videos using FIJI (NIH). Videos are representative of 3 experiments.

Measurement of chymase activity, tryptase activity, and complement C3 in mouse serum. Functional serum tryptase and chymase activities were measured by using the Mast Cell Degranulation Assay Kit (Millipore, IMM001) and the Chymase Activity Assay Kit (Sigma-Aldrich, CS1140), respectively. Following the manufacturers' instructions, the reaction was carried out in a total volume of $100 \mu \mathrm{l}$ using $5 \mu \mathrm{l}$ of serum, and then relative chymase and tryptase activity were calculated by normalizing the values to chymase and tryptase activity in the serum of mock-infected mice. Complement C3 concentrations in serum were measured using the Mouse Complement C3 ELISA Kit (Abcam, ab157711) with a minimum detection range of $3.13 \mathrm{ng} / \mathrm{ml}$ to $200 \mathrm{ng} / \mathrm{ml}$. Serum samples were diluted 1:50,000 times according to the manufacturer's instructions, and $100 \mu \mathrm{l}$ of diluted serum was used for the assay.

Detection of MC proteases in clinical samples. Human clinical samples were collected from hospitalized patients as part of surveillance for severe dengue in Jakarta, Indonesia, from 1975 to 1978 (46-48). Other than for an unknown period in the 1990s when a freezer failed and the samples thawed, they were maintained at -60 to $-80^{\circ} \mathrm{C}$ for the 4 decades of storage. The DHF grade was retrospectively classified according to the 1997 WHO classification criteria (3). For the second Sri Lankan cohort, human clinical samples were prospectively obtained by the Ministry of Health in Sri Lanka and provided by the
Dengue Tools Project $(83,84)$. Dengue Tools recruited patients with undifferentiated febrile illness with a duration of less than 7 days, and serum samples were selected for this study based on being confirmed dengue positive using previously described methods (84). Dengue Tools serum samples collected at the time of patient recruitment were selected for tryptase testing based on having had fever for less than 6 days, a clear discharge diagnosis of either DF or DHF, and having sufficient remaining serum to perform the test. Classification was also determined based on the 1997 WHO criteria (3). Serum was stored at $-80^{\circ} \mathrm{C}$. All DHF patients met the WHO case classification for grades I, II, III, or IV (3). ELISAs for human chymase (Blue Gene, E01M0368) and human tryptase (Cloud-Clone Corp., SEBO7OHu) were performed on samples in a blinded manner according to the manufacturers' instructions and unblinded only after analysis.

Statistics. Prism 7 and Excel were used to determine statistical significance, and SPSS was used to verify that the input data were normally distributed using the Shapiro-Wilk test prior to analysis using parametric tests. Violin plots were generated using a web-based tool (85). Where appropriate for direct comparisons of 2 samples, Student's unpaired $t$ test was used. For multiple groups, 1- or 2-way ANOVA was performed with post tests to determine between-groups statistical significance or regression analysis in the case of human biomarker data. Data were considered significant at $P \leq 0.05$. The numbers of biological or technical replicates for each group are indicated in each figure legend.

Study approval. Animal studies were approved by SingHealth Institutional Animal Care and Use Committee, Singapore. Ethical approval for clinical samples was obtained from the Ethics Review Committee, Faculty of Medicine, University of Colombo, Sri Lanka and the Institutional Review Board, National University of Singapore. All patients gave informed consent.

\section{Author contributions}

The project was conceived by ALSJ and APSR. Experiments were performed by APSR, CKM, SABA, AS, JO, CJJ, and CCG. Data were analyzed and interpreted by ALSJ, APSR, CKM, and DJG. The manuscript was written by APSR and ALSJ. Human clinical samples were provided by DJG, HT, and AWS. DJG also categorized samples according to dengue classification schemes. Intravital microscopy was optimized and analyzed by CKM, CCG, and LGN. All authors contributed to discussions and reviewed the manuscript.

\section{Acknowledgments}

This work was funded by the National Medical Research Council of Singapore (NMRC/CBRG/0084/2015) and received start-up funding from Duke-National University of Singapore Medical School.

Address correspondence to: Ashley St. John, Program in Emerging Infectious Diseases, Duke-National University of Singapore Graduate Medical School, 8 College Road, Level 9, Singapore. Phone: 65.9771.7231; Email: ashley.st.john@duke-nus.edu.sg. Or to: Abhay Rathore, Duke University Medical Center, Jones Building Room 252, 207 Research Dr., Durham, NC 27705, USA. Phone: 919.684.6942; Email: abhay.rathore@duke.edu.

APSR's present address is: Department of Pathology, Duke University Medical Center, Durham, North Carolina, USA. 
1. Simmons CP, Farrar JJ, Nguyen vV, Wills B. Dengue. N Engl J Med. 2012;366(15):1423-1432.

2. St John AL, Abraham SN, Gubler DJ. Barriers to preclinical investigations of anti-dengue immunity and dengue pathogenesis. Nat Rev Microbiol. 2013;11(6):420-426.

3. WHO. Dengue haemorrhagic fever: diagnosis, treatment, prevention, and control. 2nd ed. Geneva, Switzerland:WHO; 1997.

4. St John AL. Influence of mast cells on dengue protective immunity and immune pathology. PLoS Pathog. 2013;9(12):e1003783.

5. St John AL, Rathore APS. Adaptive immune responses to primary and secondary dengue virus infections. Nat Rev Immunol. 2019;19(4):218-230.

6. Shresta S, Sharar KL, Prigozhin DM, Beatty PR, Harris E. Murine model for dengue virusinduced lethal disease with increased vascular permeability. JVirol. 2006;80(20):10208-10217.

7. Singla M, et al. Correction: Immune response to dengue virus infection in pediatric patients in New Delhi, India-association of viremia, inflammatory mediators and monocytes with disease severity. PLoS Negl Trop Dis. 2016;10(4):e0004642.

8. Wang L, Chen RF, Liu JW, Yu HR, Kuo HC, Yang KD. Implications of dynamic changes among tumor necrosis factor-alpha (TNF-alpha), membrane TNF receptor, and soluble TNF receptor levels in regard to the severity of dengue infection. Am J Trop Med Hyg. 2007;77(2):297-302.

9. Chen LC, et al. Correlation of serum levels of macrophage migration inhibitory factor with disease severity and clinical outcome in dengue patients. Am J Trop Med Hyg. 2006;74(1):142-147.

10. Bokisch VA, Top FH, Russell PK, Dixon FJ, Müller-Eberhard HJ. The potential pathogenic role of complement in dengue hemorrhagic shock syndrome. N Engl JMed. 1973;289(19):996-1000.

11. Avirutnan P, et al. Vascular leakage in severe dengue virus infections: a potential role for the nonstructural viral protein NS1 and complement. J Infect Dis. 2006;193(8):1078-1088.

12. Modhiran N, et al. Dengue virus NS1 protein activates cells via Toll-like receptor 4 and disrupts endothelial cell monolayer integrity. Sci Transl Med. 2015;7(304):304ra142.

13. Beatty PR, Puerta-Guardo H, Killingbeck SS, Glasner DR, Hopkins K, Harris E. Dengue virus NS1 triggers endothelial permeability and vascular leak that is prevented by NS1 vaccination. Sci Transl Med. 2015;7(304):304ra141.

14 . Watanabe $S$, et al. The magnitude of dengue virus NS1 protein secretion is strain dependent and does not correlate with severe pathologies in the mouse infection model. J Virol. 2012;86(10):5508-5514.

15. Duyen HT, et al. Kinetics of plasma viremia and soluble nonstructural protein 1 concentrations in dengue: differential effects according to serotype and immune status. J Infect Dis. 2011;203(9):1292-1300.

16. Libraty DH, et al. High circulating levels of the dengue virus nonstructural protein NS1 early in dengue illness correlate with the development of dengue hemorrhagic fever. J Infect Dis. 2002;186(8):1165-1168.
17. Gonçalves BS, et al. Dynamics of nonstructural glycoprotein-1 in dengue patients presenting with different clinical manifestations from 1986 to 2012 in Rio de Janeiro, Brazil. J Med Virol. 2019;91(4):555-563.

18. Tricou V, Minh NN, Farrar J, Tran HT, Simmons CP. Kinetics of viremia and NS1 antigenemia are shaped by immune status and virus serotype in adults with dengue. PLoS Negl Trop Dis. 2011;5(9):e1309.

19. Valent P. Mast cell activation syndromes: definition and classification. Allergy. 2013;68(4):417-424.

20. Sricharoen P, Sittichanbuncha Y, Wibulpolprasert A, Srabongkosh E, Sawanyawisuth K. What clinical factors are associated with biphasic anaphylaxis in Thai adult patients? Asian Pac J Allergy Immunol. 2015;33(1):8-13.

21. Umetsu DT, Hahn JS, Perez-Atayde AR, Geha RS. Serum sickness triggered by anaphylaxis: a complication of immunotherapy. J Allergy Clin Immunol. 1985;76(5):713-718.

22. Hallgren J, Gurish MF. Pathways of murine mast cell development and trafficking: tracking the roots and routes of the mast cell. Immunol Rev. 2007;217:8-18.

23. Abraham SN, St John AL. Mast cell-orchestrated immunity to pathogens. Nat Rev Immunol. 2010;10(6):440-452.

24. St John AL, et al. Immune surveillance by mast cells during dengue infection promotes natural killer (NK) and NKT-cell recruitment and viral clearance. Proc Natl Acad Sci USA. 2011;108(22):9190-9195.

25. St John AL, Abraham SN. Innate immunity and its regulation by mast cells. JImmunol. 2013;190(9):4458-4463.

26. Kunder CA, St John AL, Abraham SN. Mast cell modulation of the vascular and lymphatic endothelium. Blood. 2011;118(20):5383-5393.

27. Cheng LE, Hartmann K, Roers A, Krummel MF, Locksley RM. Perivascular mast cells dynamically probe cutaneous blood vessels to capture immunoglobulin E. Immunity. 2013;38(1):166-175.

28. Wernersson S, Pejler G. Mast cell secretory granules: armed for battle. Nat Rev Immunol. 2014;14(7):478-494.

29. Röhlich P, Anderson P, Uvnäs B. Electron microscope observations on compounds 48-80-induced degranulation in rat mast cells. Evidence for sequential exocytosis of storage granules. J Cell Biol. 1971;51(21):465-483.

30. Kunder CA, et al. Mast cell-derived particles deliver peripheral signals to remote lymph nodes. JExp Med. 2009;206(11):2455-2467.

31. Reilly CF, Tewksbury DA, Schechter NM, Travis J. Rapid conversion of angiotensin I to angiotensin II by neutrophil and mast cell proteinases. J Biol Chem. 1982;257(15):8619-8622.

32. Corvera CU, et al. Mast cell tryptase regulates rat colonic myocytes through proteinase-activated receptor 2. J Clin Invest. 1997;100(6):1383-1393.

33. Mantri CK, St John AL. Immune synapses between mast cells and $\gamma \delta \mathrm{T}$ cells limit viral infection. J Clin Invest. 2019;129(3):1094-1108.

34. St John AL, Rathore AP, Raghavan B, Ng ML, Abraham SN. Contributions of mast cells and vasoactive products, leukotrienes and chymase, to dengue virus-induced vascular leakage. Elife. 2013;2:e00481.

35. Syenina A, Jagaraj CJ, Aman SA, Sridharan A, St John AL. Dengue vascular leakage is augmented by mast cell degranulation mediated by immunoglobulin Fc $\gamma$ receptors. Elife. 2015;4.

36. Tissera $\mathrm{H}$, et al. Chymase level is a predictive biomarker of dengue hemorrhagic fever in pediatric and adult patients. J Infect Dis. 2017;216(9):1112-1121.

37. Lindstedt KA, Kovanen PT. Isolation of mast cell granules. Curr Protoc Cell Biol. 2006; Chapter 3:Unit 3.16.

38. Molino $\mathrm{M}$, et al. Interactions of mast cell tryptase with thrombin receptors and PAR-2.J Biol Chem. 1997;272(7):4043-4049.

39. Kind LS. Fall in rectal temperature as an indication of anaphylactic shock in the mouse. J Immunol. 1955;74(5):387-390.

40. Jönsson F, et al. Mouse and human neutrophils induce anaphylaxis. J Clin Invest. 2011;121(4):1484-1496.

41. Vlach KD, Boles JW, Stiles BG. Telemetric evaluation of body temperature and physical activity as predictors of mortality in a murine model of staphylococcal enterotoxic shock. Comp Med. 2000;50(2):160-166.

42. Yamamoto K, Ito H, Hiraiwa T, Tanaka K. Effects of nafamostat mesilate on coagulopathy with chronic aortic dissection. Ann Thorac Surg. 2009;88(4):1331-1333.

43. Aoyama T, et al. Pharmacological studies of FUT175 , nafamstat mesilate. I. Inhibition of protease activity in in vitro and in vivo experiments. Jpn J Pharmacol. 1984;35(3):203-227.

44. Prieto-García A, et al. Mast cell restricted mouse and human tryptase-heparin complexes hinder thrombin-induced coagulation of plasma and the generation of fibrin by proteolytically destroying fibrinogen. J Biol Chem. 2012;287(11):7834-7844.

45. Fukuoka Y, Xia HZ, Sanchez-Muñoz LB, Dellinger AL, Escribano L, Schwartz LB. Generation of anaphylatoxins by human beta-tryptase from $\mathrm{C} 3, \mathrm{C} 4$, and C5. JImmunol. 2008;180(9):6307-6316.

46. Sumarmo, Wulur H, Jahja E, Gubler DJ, Suharyono W, Sorensen K. Clinical observations on virologically confirmed fatal dengue infections in Jakarta, Indonesia. Bull World Health Organ. 1983;61(4):693-701.

47. Gubler DJ, Suharyono W, Sumarmo, Wulur H, Jahja E, Sulianti Saroso J. Virological surveillance for dengue haemorrhagic fever in Indonesia using the mosquito inoculation technique. Bull World Health Organ. 1979;57(6):931-936.

48. Gubler DJ, Suharyono W, Lubis I, Eram S, Sulianti Saroso J. Epidemic dengue hemorrhagic fever in rural Indonesia. I. Virological and epidemiological studies. Am J Trop Med Hyg. 1979;28(4):701-710.

49. Payne V, Kam PC. Mast cell tryptase: a review of its physiology and clinical significance. Anaesthesia. 2004;59(7):695-703.

50. Stack MS, Johnson DA. Human mast cell tryptase activates single-chain urinary-type plasminogen activator (pro-urokinase). J Biol Chem. 1994;269(13):9416-9419.

51. Fresh JW, Reyes V, Clarke EJ, Uylangco CV. Philippine hemorrhagic fever: a clinical, laboratory, and necropsy study. J Lab Clin Med. 1969;73(3):451-458. 
52. Srichaikul T, Nimmanitaya S, Artchararit N, Siriasawakul T, Sungpeuk P. Fibrinogen metabolism and disseminated intravascular coagulation in dengue hemorrhagic fever. Am J Trop Med Hyg. 1977;26(3):525-532.

53. Wills BA, et al. Coagulation abnormalities in dengue hemorrhagic Fever: serial investigations in 167 Vietnamese children with Dengue shock syndrome. Clin Infect Dis. 2002;35(3):277-285.

54. Srichaikul T, Punyagupta S, Nitiyanant P, Alkarawong K. Disseminated intravascular coagulation in adult Dengue haemorrhagic fever: Report of three cases. Southeast Asian J Trop Med Public Health. 1975;6(1):106-114.

55. Choi JY, et al. Nafamostat mesilate as an anticoagulant during continuous renal replacement therapy in patients with high bleeding risk: a randomized clinical trial. Medicine (Baltimore). 2015;94(52):e2392.

56. Na KR, Choi H, Jeong JY, Lee KW, Chang YK, Choi DE. Nafamostat mesilate attenuates ischemia-reperfusion-induced renal injury. Transplant Proc. 2016;48(6):2192-2199.

57. Mori S, Itoh Y, Shinohata R, Sendo T, Oishi R, Nishibori M. Nafamostat mesilate is an extremely potent inhibitor of human tryptase. JPharmacol Sci. 2003;92(4):420-423.

58. Chen CL, et al. Serine protease inhibitors nafamostat mesilate and gabexate mesilate attenuate allergen-induced airway inflammation and eosinophilia in a murine model of asthma. JAllergy Clin Immunol. 2006;118(1):105-112.

59. Ui H, Andoh T, Lee JB, Nojima H, Kuraishi Y. Potent pruritogenic action of tryptase mediated by PAR-2 receptor and its involvement in anti-pruritic effect of nafamostat mesilate in mice. Eur J Pharmacol. 2006;530(1-2):172-178.

60. Kozik A, Moore RB, Potempa J, Imamura T, Rapala-Kozik M, Travis J. A novel mechanism for bradykinin production at inflammatory sites. Diverse effects of a mixture of neutrophil elastase and mast cell tryptase versus tissue and plasma kallikreins on native and oxidized kininogens. J Biol Chem. 1998;273(50):33224-33229.

61. Becker BF, Jacob M, Leipert S, Salmon AH, Chappell D. Degradation of the endothelial glycocalyx in clinical settings: searching for the sheddases. Br J Clin Pharmacol. 2015;80(3):389-402.

62. Tang TH, et al. Increased serum hyaluronic acid and heparan sulfate in dengue fever: association with plasma leakage and disease severity. Sci Rep. 2017;7:46191

63. Suwarto S, Sasmono RT, Sinto R, Ibrahim E, Suryamin M. Association of endothelial glycocalyx and tight and adherens junctions with severity of plasma leakage in dengue infection. JInfect Dis. 2017;215(6):992-999.

64. Lohi J, Harvima I, Keski-Oja J. Pericellular substrates of human mast cell tryptase: 72,000 dalton gelatinase and fibronectin. JCell Biochem. 1992;50(4):337-349.

65. Huang C, et al. The tryptase, mouse mast cell protease 7 , exhibits anticoagulant activity in vivo and in vitro due to its ability to degrade fibrinogen in the presence of the diverse array of protease inhibitors in plasma.J Biol Chem. 1997;272(50):31885-31893.

66. King CA, Anderson R, Marshall JS. Dengue virus selectively induces human mast cell chemokine production. JVirol. 2002;76(16):8408-8419.

67. Appanna R, Wang SM, Ponnampalavanar SA, Lum LC, Sekaran SD. Cytokine factors present in dengue patient sera induces alterations of junctional proteins in human endothelial cells. Am J Trop Med Hyg. 2012;87(5):936-942.

68. Wypij DM, Nichols JS, Novak PJ, Stacy DL, Berman J, Wiseman JS. Role of mast cell chymase in the extracellular processing of big-endothelin-1 to endothelin-1 in the perfused rat lung. Biochem Pharmacol. 1992;43(4):845-853.

69. Hsieh JT, Rathore APS, Soundarajan G, St John AL. Japanese encephalitis virus neuropenetrance is driven by mast cell chymase. Nat Commun . 2019;10(1):706

70. Vartio T, Seppä H, Vaheri A. Susceptibility of soluble and matrix fibronectins to degradation by tissue proteinases, mast cell chymase and cathepsin G. J Biol Chem. 1981;256(1):471-477.

71. Martínez Gómez JM, et al. Maternal antibody-mediated disease enhancement in type i interferon-deficient mice leads to lethal disease associated with liver damage. PLoS Negl Trop Dis. 2016;10(3):e0004536.

72. Ferreira RA, et al. Circulating cytokines and chemokines associated with plasma leakage and hepatic dysfunction in Brazilian children with dengue fever. Acta Trop. 2015;149:138-147. 73. Schwartz LB, Bradford TR. Regulation of trypt- ase from human lung mast cells by heparin. Stabilization of the active tetramer. J Biol Chem. 1986;261(16):7372-7379.

74. Pemberton AD, Huntley JF, Miller HR. Differential inhibition of mast cell chymases by secretory leukocyte protease inhibitor. Biochim Biophys Acta.1998;1379(1):29-34.

75. Welle M. Development, significance, and heterogeneity of mast cells with particular regard to the mast cell-specific proteases chymase and tryptase. J Leukoc Biol. 1997;61(3):233-245.

76. Low JG, et al. Early dengue infection and outcome study (EDEN) - study design and preliminary findings. Ann Acad Med Singap. 2006;35(11):783-789.

77. Saleh R, et al. A new human mast cell line expressing a functional IgE receptor converts to tumorigenic growth by KIT D816V transfection. Blood. 2014;124(1):111-120.

78. Hallgren J, Pejler G. Biology of mast cell tryptase. An inflammatory mediator. FEBS J. 2006;273(9):1871-1895.

79. Watanabe S, Chan KW, Dow G, Ooi EE, Low JG, Vasudevan SG. Optimizing celgosivir therapy in mouse models of dengue virus infection of serotypes 1 and 2: The search for a window for potential therapeutic efficacy. Antiviral Res. 2016;127:10-19.

80. Egawa G, Nakamizo S, Natsuaki Y, Doi H, Miyachi Y, Kabashima K. Intravital analysis of vascular permeability in mice using two-photon microscopy. Sci Rep. 2013;3:1932.

81. Li JL, et al. Intravital multiphoton imaging of immune responses in the mouse ear skin. Nat Protoc. 2012;7(2):221-234.

82. Schindelin J, et al. Fiji: an open-source platform for biological-image analysis. Nat Methods. 2012;9(7):676-682.

83. Wilder-Smith A, et al. DengueTools: innovative tools and strategies for the surveillance and control of dengue. Glob Health Action. 2012;5.

84. Tissera $\mathrm{H}$, et al. Laboratory-enhanced dengue sentinel surveillance in Colombo District, Sri Lanka: 2012-2014. PLoS Negl Trop Dis. 2016;10(2):e0004477.

85. Weissgerber TL, Savic M, Winham SJ, Stanisavljevic D, Garovic VD, Milic NM. Data visualization, bar naked: A free tool for creating interactive graphics. J Biol Chem. 2017;292(50):20592-20598. 